\title{
Multi-Dimensional Dynamics of Human Electromagnetic Brain Activity
}

\author{
Tetsuo Kida *, Emi Tanaka and Ryusuke Kakigi \\ Department of Integrative Physiology, National Institute for Physiological Sciences, Okazaki, Japan
}

Magnetoencephalography (MEG) and electroencephalography (EEG) are invaluable neuroscientific tools for unveiling human neural dynamics in three dimensions (space, time, and frequency), which are associated with a wide variety of perceptions, cognition, and actions. MEG/EEG also provides different categories of neuronal indices including activity magnitude, connectivity, and network properties along the three dimensions. In the last 20 years, interest has increased in inter-regional connectivity and complex network properties assessed by various sophisticated scientific analyses. We herein review the definition, computation, short history, and pros and cons of connectivity and complex network (graph-theory) analyses applied to MEG/EEG signals. We briefly describe recent developments in source reconstruction algorithms essential for source-space connectivity and network analyses. Furthermore, we discuss a relatively novel approach used in MEG/EEG studies to examine the complex dynamics represented by human brain activity. The correct and effective use of these neuronal metrics provides a new insight into the multi-dimensional dynamics of the neural representations of various functions in the complex human brain.

OPEN ACCESS

Edited by: Mikhail Lebedev,

Duke University, USA

Reviewed by:

Hiroki Nakata

Nara Women's University, Japan

Selma Supek,

University of Zagreb, Croatia

*Correspondence:

Tetsuo Kida

nikita@nips.ac.jp

Received: 27 October 2015 Accepted: 21 December 2015

Published: 19 January 2016

Citation:

Kida T, Tanaka E and Kakigi R (2016)

Multi-Dimensional Dynamics of

Human Electromagnetic Brain Activity.

Front. Hum. Neurosci. 9:713

doi: 10.3389/fnhum.2015.00713
Keywords: inter-regional connectivity, graph theory, complex systems, non-linear dynamical system, neuronal avalanche, self-organized criticality

\section{INTRODUCTION}

Neuroscience has been witness to a long-standing debate on functional specialization (or localization) of the human brain since the early times of Franz Josef Gall, Marie-Jean-Pierre Flourens, Paul Broca, John Hughlings Jackson, and other individuals of note in this field. Pioneering research, which examined single-neuronal activity using micro-electrodes in conscious monkeys (Evarts, 1968; Hubel and Wiesel, 1968; Bruce et al., 1981) and human patients (Quiroga et al., 2005), revealed that neuronal activity patterns differed depending on brain regions, thereby providing evidence for functional specialization. Recordings of electric potentials from several regions of the brain (Allison et al., 1991, 1999) and their stimulation in humans and animals (Penfield and Rasmussen, 1950; Parvizi et al., 2012) further supported functional specialization. Functional magnetic resonance imaging (fMRI) (Ogawa et al., 1990; Bandettini et al., 1992; Friston et al., 1994) has also markedly accelerated the concept of functional specialization. Previous studies have identified a large body of representations in the brain, e.g., for retinotopy (Sereno et al., 1995; Wandell et al., 2007), tonotopy (Langers et al., 2007), somatotopy (Martuzzi et al., 2014), multisensations (Downar et al., 2000; Beauchamp et al., 2004), the face (Puce et al., 1996; Kanwisher et al., 1997), body parts (Downing et al., 2001), mirror system (Iacoboni et al., 2005), the theory 
of the mind (Gallagher et al., 2000), and even love (Bartels and Zeki, 2004), in association with fMRI studies on non-human primates (Tsao et al., 2006, 2008). Furthermore, lesion studies on human patients (Vaina, 1989; Damasio et al., 1994) and activation studies using positron emission tomography (PET) (Roland et al., 1980; Corbetta et al., 1990) have also provided essential evidence for functional specialization. Magnetoencephalography (MEG) has also demonstrated functional specialization in sensory cortices by estimating somatotopy (Baumgartner et al., 1991), tonotopy (Romani et al., 1982; Pantev et al., 1989), and retinotopy (Aine et al., 1996; Supek et al., 1999). Thus, the idea of functional specialization in non-invasive neuroimaging has been examined extensively and is now largely accepted. In these fMRI, PET, MEG, and EEG studies, the spatial patterns of differences in activation between a task and the baseline, and between different tasks or stimuli have been one of the most common sources of evidence for functional specialization. We herein refer to this type of activity difference as the first category of neural indices. Virtual lesion studies using transcranial magnetic stimulation (TMS) have been another crucial source for functional specialization.

Individual brain regions have billions of reciprocal structural connections, and send neuronal signals to each other in the form of action potentials. This type of reciprocal information transfer may directly or indirectly change temporal patterns or phase differences between population-level neural signals recorded from different brain regions using neuroimaging techniques. Therefore, unlike the functional separation of brain regions, signal transmission or information transfer between brain regions cannot be assessed based only on differences in the magnitude of population-level neuronal or hemodynamic activation. Inter-regional connectivity may have the capacity to estimate cortical information transfer, and is computed from structural and anatomical brain signals in various manners. Connectivity includes structural and functional connectivities, which are obtained by analyzing anatomical MR signals and functional neuroimaging signals, respectively. Structural or anatomical connectivity is obtained using diffusion spectral imaging (DSI) (Hagmann et al., 2007, 2008), diffusion tensor imaging (DTI), and cortical thickness (Iturria-Medina et al., 2007), whereas functional connectivity was initially defined as temporal correlations between spatially remote neurophysiological events in an early PET study (Friston et al., 1993b; Friston, 1994). Thus, functional connectivity itself provides no directed information, i.e., no information on the causality of a relationship, whereas effective connectivity typically comprises an estimate of directed influence between brain regions (Friston et al., 1993a). These concepts originated from multiunit electrode recordings (Gerstein and Perkel, 1969; Gerstein et al., 1989; Aertsen and Preissl, 1991; Gochin et al., 1991). Many metrics for functional and effective connectivities are currently computed from brain signals measured using various sophisticated recording techniques including fMRI, electroencephalography (EEG), MEG, nearinfrared spectroscopy (NIRS), electrocorticography (ECoG), and local field potential (LFP). We herein defined connectivity as the second category, and focused on MEG/EEG connectivity.
The third category is based on an advanced and integrated view of connectivity, and is computed using a complex network or graph-theoretical analysis (Watts and Strogatz, 1998; Barabasi and Albert, 1999; Bullmore and Sporns, 2009; Sporns and Bullmore, 2014; Stam, 2014). Individual brain regions or neurons (nodes or vertices) are activated through neuronal connections (links or edges), and function locally and globally as a network to realize complex human perceptions, cognition, and actions. Connectivity is the strength or degree of the relationship between signals from two or more brain regions (or neurons), while graph metrics describe the various local and global properties of a network by taking many connectivity values into consideration. These three categories of neuronal metrics derived from multichannel, rapidly time-varying brain signals provide complementary information on each other. In recent years, marked advances have been made in the latter two categories, connectivity and graph metrics, which we herein review. The connectivity and graph-theoretical analyses also lead to a rediscovery and extension of the idea of functional specialization in terms of network and its elements. We will briefly describe the basics of MEG/EEG, followed by an overview of connectivity and graph metrics. We will then provide a short overview on another recent topic in human neuroscience regarding dynamical and complex systems, which has been attracting interest. Experts on MEG/EEG may skip the next section "Basics of MEG/EEG" and proceed to the sections "Connectivity" and "Network metrics."

\section{BASICS OF MEG/EEG}

MEG is an invaluable functional brain imaging technique that provides direct, real-time monitoring of neuronal activity with high temporal resolution (Cohen, 1968, 1972; Hämäläinen et al., 1993; Kakigi et al., 2000; Supek and Aine, 2014; Hari and Parkkonen, 2015). The cerebral cortex in the human brain is a 2-4-mm-thick sheet of gray matter that generally has 5 or 6 layers. Pyramidal neurons are aligned in the same direction perpendicularly along the surface of the cortex, in which there are at least $10^{10}$ neurons. When large numbers of neurons with the same orientation in restricted cortical layers are synchronously activated by trans-synaptic inputs, it is possible to detect the resulting magnetic fields using magnetic field sensors placed near the scalp. These trans-synaptic inputs induce a primary current, which is related to the movement of ions due to their chemical concentration gradients, and passive ohmic current (also called volume current), which occurs in the surrounding medium, in the brain as a volume conductor, with the latter current completing the loop of ionic flow in order to prevent the buildup of charge in the conductor (Hämäläinen et al., 1993). Although magnetic fields are generated from primary and volume currents in the brain, the volume current does not produce any magnetic fields outside a symmetric volume conductor, and, thus, contributes less to the extracranially-recorded magnetic field. Radial current sources also do not produce any magnetic fields outside a symmetric volume conductor, whereas tangential current sources do. Therefore, MEG is advantageous for recording activity 
from the cortical sulci, but may also measure magnetic fields originating from cortical gyri to a certain extent because no cortices are oriented completely perpendicular to the scalp or brain surface (to the surface of sensors) (Hillebrand and Barnes, 2002; Ahlfors et al., 2010). EEG records the time series of a potential difference (voltage) between two sites (recording and reference sites). EEG signals mainly include volume currents originating from both radial and tangential current sources. EEG is a classical technique that has been used for approximately 100 years since its first application to humans (Berger, 1929). Recent advances in multichannel recordings, signal processing, portability, multimodal recordings, and multi-person recordings have rendered EEG a unique and powerful neuroimaging tool. Although the spatial resolution of EEG is sometimes regarded as being inferior to that of MEG, proper sampling and the correct analysis of electric fields provide reliable information on neuronal activity with high temporal resolution (Michel and Murray, 2012).

The most important advantage of MEG/EEG is excellent temporal resolution in the order of milliseconds. Temporal resolution may be considered in two manners; one is as an aspect of the measuring device, while the other is as a physiological aspect depending on its electromagnetic properties and the temporal profile of underlying neuronal activity. This advantage also enables examinations in a wide range of frequencies from near-DC to more than $100 \mathrm{~Hz}$ by transforming time-dimension data to frequency-dimension data with, for example, Fourier, Wavelet, Stockwell and Hilbert analyses, which provides two-dimensional neural representations in time and frequency in different brain regions (time-frequency representation, TFR). Moreover, it is possible to consider a three-dimensional representation by adding a space dimension to TFR, thereby extending it to a space-time-frequency representation (STFR). In principle, increasing the dimension of the representation is possible by considering different aspects of datasets, e.g., experimental variables; however, we herein focus on these three dimensions. These features, which are based on high temporal resolution and multichannel recording, provide detailed information on the cortical mechanisms underlying perceptions, cognition, and actions, which may be hidden in MEG and EEG data.

MEG and EEG signals themselves are recorded from sensors or electrodes, and, thus, are estimated in the source-space using various source estimation techniques with different constraints. These include an equivalent current dipole (ECD) source estimation (Sarvas, 1987; Hämäläinen et al., 1993), minimum L2 norm estimation (MNE) (Hämäläinen and Ilmoniemi, 1994), minimum current (L1 norm) estimation (MCE) (Uutela et al., 1999), and beamforming (Robinson and Varba, 1999; Gross et al., 2001; Sekihara et al., 2001). In the ECD estimation, activity is assumed to be focal, and may be explained by a small number of current dipoles. MNE is a type of distributed source modeling, which divide the source space into a grid that has a large number of dipoles (between hundreds and thousands) over the cortical surface and estimate the current in all modeled source locations at each time point with minimum power (L2-norm), resulting in data that is consistent with the measurement. MCE provides more focal estimates by using an L1-norm prior to those provided by MNE. Thus, MCE and MNE are suitable for analyzing evoked responses and examining widespread activation over time. Beamforming is also a type of distributed source modeling that acts as a spatial filter to pass brain activity from a specified location and attenuate activity from other locations. Time-domain and frequency-domain implementations of beamforming exist, e.g., a linear-constraint minimum variance beamformer (LCMV) and the dynamic imaging of coherent sources (DICS), respectively. Low-resolution electromagnetic tomography (LORETA) and its variants (e.g., eLORETA) are another family of distributed source modeling, and estimate current distributions with zero localization errors by using sparse (low spatial resolution) source configuration. Distributed source models have the advantage of no prior assumption of the source model being necessary. Recent advances in source estimation techniques have suggested a range of new algorithms as well as extended versions of early algorithms (Diwakar et al., 2011; Owen et al., 2012; Babadi et al., 2014; Huang et al., 2014; Mohseni et al., 2014). Various source estimation algorithms will be reviewed in conjunction with computations of connectivity metrics in a later section.

The brain (anatomy) has to be divided into different regions, which are used to set dipoles (or virtual sensors) for estimations of signal sources and the subsequent computation of connectivity and graph metrics. This brain parcellation may be performed on the basis on an anatomical atlas (structural parcellation) and functionally-separated regions (functional parcellation). Typical anatomical atlases include Talairach, AAL, and HarvardOxford atlases, whereas functional parcellation has recently been suggested for resting-state fMRI studies (Power et al., 2011; Craddock et al., 2012). Previous MEG studies used anatomical atlases to compute graph metrics (Jin et al., 2013, 2014). Another method to set dipoles within the brain is not based on any atlas. In this case, we set more dipoles with arbitrary spacing within the brain after cortical segmentation in the beamforming technique or set dipoles on cortical meshes in the minimum norm or current estimation technique. Once it has been decided how the brain is to be divided, dipoles are placed on each ROI and source estimations are then performed. Connectivity and graph metrics may also be computed from sensor-space MEG signals; however, source-space data may be more appropriate for avoiding spurious connectivity caused by a common signal source and establishing what brain regions are involved in changes in connectivity and network properties.

\section{CONNECTIVITY METRICS}

Various types of metrics may be used to quantify functional and effective connectivities for MEG/EEG signals. These metrics are used to examine relationships between two time series experimentally recorded from, for example, different brain regions, from a brain region and muscle, from cortical and subcortical regions, from a subcortical region and muscle (i.e., electromyographic activity), and from a cortical region and kinematics (i.e., acceleration of movement). These metrics also differ in linearity, the information included (amplitude and 
phase), volume conduction/field spread-free, and directionality (i.e., functional or effective). In the early half of this section, we provide an overview of the definition, computation, short history, and experimental comparisons of these functional connectivity metrics, and then review effective connectivity metrics.

\section{FUNCTIONAL CONNECTIVITY}

\section{Correlation}

A correlation refers to a broad class of statistical relationships involving interdependence, which is any statistical relationship between two random variables or two datasets (corresponding to two time series in neuroimaging datasets). In fMRI studies, functional connectivity is an observable phenomenon that is quantifiable with measures of statistical dependencies, such as correlations and coherence (Friston, 1994). In the EEG community, correlations were frequently used to examine relationships between two EEG signals (Adey et al., 1961; Walter, 1963) even before functional and effective connectivities were introduced to fMRI data (Friston, 1994). However, at that time, the spatial blurring of electrical activity due to volume conduction and reference electrodes was already an important challenge when using correlations between two EEG time series as a metric of interregional dependence, as with coherence which is described in the next section. Electric fields generated in the brain spread in space via different conductor media (volume conduction), and, thus, EEG signals recorded by different scalp sites include an electric field derived from a common current source. Furthermore, recordings of small electric fields from the human brain require differential amplification, which needs a common, reference potential as an input. These two issues lead to a common difficulty in interpreting correlations between EEG signals. The increased strength of a common signal source obscures true interdependence between two signals recorded from two different sites. In MEG, magnetic fields are hardly affected by the conductor medium and MEG signals from different channels are not associated with any reference signal derived from a common signal source in the brain. Therefore, MEG is superior to EEG but MEG signals may still share a common cortical source (called field spread), even for planar gradiometers, despite the influence being less than in EEG. One strategy to overcome this spurious correlation is to project sensor-space data to the source space; however, signal leakage, which is an apparent signal leak between voxels in the brain space, remains due to the ill-posed nature of the inverse problem, inaccuracies in the forward solution, and incorrect assumptions caused by the inverse localization algorithm used (Brookes et al., 2014). Accordingly, correlations are generally not considered to be the best metric for examining functional connectivity in EEG and MEG, except for specific cases, e.g., distant connectivity between the cortex and another part of the body such as muscle (discussed later), the computation of correlations after specific computation (discussed later), and connectivity between the brains of different participants (hyper-scanning) (Babiloni et al., 2006; Babiloni and Astolfi, 2014; Zhdanov et al., 2015). Another important point to consider is that this issue is also inevitable to a greater or lesser extent to any non-invasive and invasive neuroimaging techniques, including fMRI, PET, NIRS, ECoG, intracerebral recordings, and LFP recordings from local regions, because signals measured by these techniques also spread in space across voxels, electrodes, and probes as in EEG and MEG.

\section{Coherence}

Coherence is a statistic representing the relationship between two signals and is also an extension of correlation to frequency domain. Coherence (magnitude-squared coherence) is defined as:

$$
C_{x y}(f)=\frac{\left|G_{x y}(f)\right|^{2}}{G_{x x}(f) G_{y y}(f)}
$$

$x(t)$ and $y(t)$ are two time series, $G_{x y}(f)$ is the cross-spectral density between $x$ and $y$, and $G_{x x}(f)$ and $G_{y y}(f)$ are the autospectral densities of $x$ and $y$, respectively. Cross-spectra density (CSD) is calculated in the frequency domain as:

$$
C_{x y}(f)=X(f) Y^{* T}(f)
$$

Thus, CSD is the complex conjugate product of Fouriertransformed data $X(f)$ and $Y(f)$. In EEG, coherence was used once (Adey et al., 1961; Walter, 1963; Sklar et al., 1972; Busk and Galbraith, 1975), but has been criticized, similar to correlations, due to issues associated with volume conduction and reference electrodes (Fein et al., 1988; Nolte et al., 2004; Nunez and Srinivasan, 2006; Stam et al., 2007b). However, coherence is applicable to some types of studies. For example, coherence has been widely and successfully used to measure functional connectivity in MEG since the introduction of the DICS method (Gross et al., 2001). Coherence is a reliable metric of functional connectivity between distant signal sources such as the cortex and muscle (Gross et al., 2001, 2005). In this case, electromyographic data recorded from peripheral muscles are used as time-series data to compute coherence. This has recently been extended to the examination of coherence between kinematics (acceleration of movement) and MEG signals (Bourguignon et al., 2013; Marty et al., 2015). Another example is demonstrated in the spino-muscle coherence of LFP in the spinal cord and electromyography in the hand muscles of conscious monkeys performing a precision motor task (Takei and Seki, 2008). A previous study investigated cortico-thalamic coherence between MEG and sub-thalamic nucleus LFP signals recorded from deep brain stimulation patients (Sander et al., 2010). It is important to note that, even for MEG, coherence may cause false positives due to the virtual zero-lag correlations caused by source leakage in functional connectivity between local regions (Brookes et al., 2014).

\section{Partial Coherence}

As described above, coherence (magnitude-squared coherence) between EEG signals is contaminated by volume conduction and reference electrodes (Rappelsberger, 1989; Andrew and Pfurtscheller, 1996; Florian et al., 1998; Mima et al., 2000). 
Partial coherence is computed by removing the linear effect of a common signal, as follows:

$$
\left|R_{x y / z}(f)\right|^{2}=\frac{\left|G_{x y}(f)-\frac{G_{x z}(f) G_{z y}(f)}{G(f)}\right|^{2}}{\left(G_{x x}(f)-\frac{\left|G_{x z}(f)\right|^{2}}{G_{z z}(f)}\right)\left(G_{y y}(f)-\frac{\left|G_{z y}(f)\right|^{2}}{G_{x x}(f)}\right)}
$$

where $G x x, G y y$, and $G z z$ are the autospectra of EEG signals, $x, y$, and $z$ for a given frequency $f$ and $G x y, G z y$, and $G x z$ are cross-spectra between two of them. Partial coherence ranges between 0 (no association) and 1 (perfect linear association). In this computation, the signal $z$ functions as a linear effect to be removed. Therefore, the signal $z$ is regarded as a good reflection of a common signal that causes virtual zero-lag coherence; if not, this metric is non-sensical. Accordingly, the signal $z$ must be selected from the recorded signals, such that it includes the signal derived from a common source, but not other relevant signals involved in true functional connectivity. For example, the signal from the occipital electrode may be used for $z$ when examining motor-related areas in the frontal lobe. However, it is important to note that, practically, there are no ideal signals for $z$ because signals from any recording sites may easily include other relevant signals that must not be removed.

\section{Phase Coherence}

An important limitation of coherence (magnitude-squared coherence) is that it only measures linear relationships between two time series and may fail to detect non-linear interdependencies between underlying dynamic systems. One possibility to overcome this issue is based on the analytical signal concept. In this approach, the instantaneous phase of time series is computed, and interactions are estimated by timedependent $n: m$ phase synchronization. Phase coherence is a statistical measure for phase synchronization. Synchronization was introduced to physics by Huygens in the 17th century for coupled oscillators. The concept of phase synchronization was introduced for coupled chaotic models (Rosenblum et al., 1996) and demonstrated experimentally (Parlitz et al., 1996). Phase coherence was introduced to represent the phase synchronization of EEG signals recorded from epileptic patients (Mormann et al., 2000) and is defined as:

$$
R=\left|\frac{1}{N} \sum_{j=0}^{N-1} e^{i \varphi_{1,1}(j \Delta t)}\right|=1-C V
$$

where $1 / \Delta t$ is the sampling rate of time series and $C V$ denotes the circular variance of an angular distribution obtained by transforming relative phase angles onto the unit circle in the complex plane. This metric is regarded as a specific case in which the phase locking ratio, $n: m$, is $1: 1$, and is applicable to investigations of $n$ : $m$ synchronization by changing $\varphi_{1,1}$ into $\varphi_{n, m}$. The use of Euler's formula changes the above equation into:

$$
R=\left|\left[\frac{1}{N} \sum_{j=0}^{N-1} \sin \left[\varphi_{1,1}(j \Delta t)\right]\right]^{2}+\left[\frac{1}{N} \sum_{j=0}^{N-1} \cos \left[\varphi_{1,1}(j \Delta t)\right]\right]^{2}\right|^{1 / 2}
$$

where $R$ ranges between 0 and 1 . Strict phase locking results in $R=1$, whereas a uniformed distribution of phases results in $R=0$. A previous MEG study computed the phase coherence of two remote source activities in order to demonstrate the attentional modulation of inter-regional synchronization (Siegel et al., 2008). This study estimated source-level coherence with a two-dipole beamforming technique that allows the reliable estimation of source-level coherence, even under conditions of very high source correlation (Schoffelen et al., 2008).

\section{Generalized Synchronization}

The above approach based on the analytical signal concept is only valid if time series are approximately oscillatory, and the theory of non-linear dynamical systems provides a more general approach. The concept of generalized synchronization was introduced by Rulkov and colleagues in the context of unidirectionally coupled driver response systems (Rulkov et al., 1995). Generalized synchronization exists between two dynamical systems, $\mathrm{X}$ and $\mathrm{Y}$, when the state of the response system, $Y$ is expressed as a function of the state of the driving system, $X$, as follows:

$$
Y=F(X)
$$

In generalized synchronization, time series do not need to resemble each other. Several algorithms have been proposed to detect this type of interdependency in experimental time-series data; mutual false nearest neighbors (Rulkov et al., 1995), its elaborated version (Schiff et al., 1996), and Arnhold's measure (Arnhold et al., 1999). However, these metrics may be biased by the properties of individual dynamic systems (Pereda et al., 2001). Therefore, synchronization likelihood (SL) was proposed to overcome this bias (Stam and Van Dijk, 2002), and is a metric sensitive to linear and non-linear synchronization. It is also suitable for analyzing non-stationary data because it is computed in a time-dependent manner. Assuming a time series of measurements $x$ and $y(i=1, \ldots, N)$ recorded from $X$ and $Y$, vectors are reconstructed in the state space of $X$ and $Y$ with time-delay embedding, as follows:

$$
\begin{aligned}
X_{i} & =\left(x_{i}, x_{i+l}, x_{i+2 l}, \ldots, x_{i+(m-1) l}\right) \\
Y_{i} & =\left(y_{i}, y_{i+l}, y_{i+2 l}, \ldots, y_{i+(m-1) l}\right)
\end{aligned}
$$

where $l$ is the lag and $m$ is the embedding dimension. The distance between two vectors in state space is a metric of their similarity. SL $S$ between $X$ and $Y$ is then defined at time $i$, as follows:

$$
S_{i}=\frac{1}{N} \sum_{j} \theta\left(\varepsilon_{y}-\left|Y_{i}-Y_{j}\right|\right) \text { if }\left(X_{i}-X_{j}<\varepsilon_{x}\right)
$$

where we only sum over those $j$ satisfying $w 1<|i-j|<w 2$, and $X_{i}-X_{j}<\varepsilon_{x} . N$ is the number of $j$ fulfilling these conditions. $w_{1}$ is the Theiler correction for autocorrelation effects and must be at least in the order of the autocorrelation time, $w_{2}$ is a window that sharpens the time resolution of the synchronization metric and is set to the range of $w_{1} \ll w_{2} \ll N$. Thus, SL is the likelihood that the recurrence of a pattern in time series $X$ at two times $i$ 
and $j$ will coincide with the recurrence of patterns in time series $Y$ at the same times $i$ and $j$, and takes on values between $\mathrm{p}_{\mathrm{ref}}$ (no coupling) and 1 (complete coupling). $\mathrm{P}_{\text {ref }}$ is a parameter of the computation of SL, and is typically set to 0.01 . Several variations of SL may be derived by averaging over time, space, or both. SL has been applied to EEG signals during movement observation (Calmels et al., 2010), movement execution (Calmels et al., 2008), and attention (Gootjes et al., 2006), and resting-state MEG signals for examinations of various diseases such as Alzheimer's disease (Stam et al., 2006, 2007a) or a subsequent graph-theory analysis (Stam, 2004).

\section{Phase Locking Value}

Coherence may not be a pure measure of the phase relationship because it contains amplitude and phase information. The phase locking value (PLV) was introduced to detect frequency-specific transient phase locking independent of amplitude (Lachaux et al., 1999). PLV is calculated by performing a bandpass filter to extract two time series at a frequency of interest, computing its convolution (its TFR) by Wavelet or Stockwell transformation and the phase difference at each time, $\theta(t)=\varphi_{x}(t)-\varphi_{y}(t)$, and is then given by:

$$
P L V=\frac{1}{T} \sum_{t}^{T} \exp (i \theta(t))
$$

$\varphi_{x}(t)$ and $\varphi_{y}(t)$ are the phase differences of time series $x(t)$ and $y(t)$. PLV calculated here reflects a single-trial phase difference, but may also be computed across trials as an average value, as follows:

$$
P L V=\frac{1}{N} \sum_{n}^{N} \exp (j \theta(t, n))
$$

where $n$ indexes the $N$ trials and $\theta(t, n)$ is the phase difference $\varphi_{1}(t, n)-\varphi_{2}(t, n)$. Note that PLV is still sensitive to volume conduction/field spread and reference electrodes.

\section{Imaginary Component of Coherency}

The imaginary component of coherency (iCoh) was introduced in an EEG study (Nolte et al., 2004) in order to overcome spurious zero-lag coherence. The complex coherency between two time series may be defined as the cross spectrum divided by the product of the two power spectra. Its mean over all frequencies may alternatively be computed via the mean over time of the corresponding analytical signals, as follows:

$$
c=\frac{\left\langle A_{1} A_{2} e^{i \Delta \phi}\right\rangle}{\sqrt{\left\langle A_{1}^{2}\right\rangle\left\langle A_{2}^{2}\right\rangle}}
$$

where $A_{1}$ and $A_{2}$ are the amplitudes of the two time series, and $\Delta \phi$ is the instantaneous phase difference between (the Hilbert transforms of) the two time series. The absolute value of coherency, typically referred to as coherence, is bounded between 0 and 1 . iCoh here is given by:

$$
\operatorname{Im}\{c\}=\frac{\left\langle A_{1} A_{2} \sin \Delta \varphi\right\rangle}{\sqrt{\left\langle A_{1}^{2}\right\rangle\left\langle A_{2}^{2}\right\rangle}}
$$

An important property of iCoh is that its (non-vanishing) finite value cannot be caused by the linear mixing of an uncorrelated source (volume conduction and reference electrode) and, thus, reflects true interactions in the source underlying the two time series. Note that iCoh depends on the amplitudes of the signals and the magnitude of the phase delay. Recent MEG studies provided evidence for the feasibility of iCoh and a relevant metric in an MEG connectivity analysis by stimulations and experiments (Sekihara et al., 2011; Ewald et al., 2012; Sekihara and Nagarajan, 2013; Cho et al., 2015; Hsiao et al., 2015).

\section{Phase Lag Index}

The phase lag index (PLI) is a metric of the asymmetry of the distribution of phase differences between two signals. This metric was introduced by Stam et al. (2007b), and removes the effect of amplitude information included in iCoh. An advantage of PLI is that it is affected less by the influence of common sources due to volume conduction and/or active reference electrodes, similar to iCoh. This metric is based on the existence of a consistent, non-zero phase lag between two time series not explainable by volume conduction from a single source, and, as a consequence, renders true interactions between the underlying systems likely. Therefore, PLI may be considered to be the same to PLV as iCoh is to coherence (Brookes et al., 2014). Such consistent, non-zero phase lags may be determined from the asymmetry of the distribution of instantaneous phase differences between two signals.

$$
P L I=\left|\left\langle\operatorname{sign}\left[\triangle \phi\left(t_{k}\right)\right]\right\rangle\right|
$$

PLI ranges between 0 and 1 . A PLI of zero indicates either no coupling or coupling with a phase difference centered around 0 and $\pi$. A PLI of 1 indicates perfect phase locking at a value of $\Delta \phi$, different from $0 \bmod \pi$. The stronger this non-zero phase locking is, the larger PLI will be. PLI has no information about the leading phase. However, this information is easily recovered by disregarding the absolute value in the equation above. Surrogate data may be introduced to determine whether PLI is significantly larger than zero. In brief, PLI has to be computed for both the original time series of a set of surrogate data that match the original data, but lacks any correlations between channels, e.g., by shifting each channel by some random phase. Differences between the PLI of original and surrogate data yield z-scores that are sufficient for defining significance levels. It is important to note that the equation of PLI requires the phase difference to be bounded in the interval $-\pi<\Delta \phi<\pi$. In contrast, if phases are defined in the interval $0<\Delta \phi \leq 2 \pi$, then the equation needs to be modified to:

$$
P L I=\left|\left\langle\operatorname{sign}\left[\sin \triangle \phi\left(t_{k}\right)\right]\right\rangle\right|
$$

\section{weighted PLI (wPLI) and Debiased wPLI (dwPLI)}

The PLI of sensitivity to noise and volume conduction may be hindered by discontinuity because perturbations turn phase lags into leads and vice versa. This has more serious implications for the synchronization effects of a small magnitude. Therefore, 
weighted PLI was developed in order to increase the capacity to detect true changes in phase synchronization, reduce the influence of common noise sources, and reduce the influence of changes in the phase of coherency (Vinck et al., 2011). In weighted PLI, the contribution of the observed phase leads and lags is weighted by the magnitude of the imaginary component of the cross-spectrum.

$$
\Phi \equiv \frac{|\{\mathfrak{I}\{X\}\}|}{E\{|\mathfrak{I}\{X\}|\}}=\frac{|E\{|\mathfrak{I}\{X\}| \operatorname{sgn}(\mathfrak{I}\{X\})\}|}{E\{|\mathfrak{I}\{X\}|\}}
$$

The numerators of wPLI and magnitude of iCoh are the same, with the only difference being normalization in the denominator. Hence, wPLI has 2 advantages over PLI; one is a reduction in sensitivity to additional, uncorrelated noise sources, while the other is an increase in the statistical power to detect changes in phase synchronization. Furthermore, the debiased version of wPLI (dwPLI) has been suggested to reduce sample-size bias, which is another factor affecting phase synchronization. dwPLI is defined as:

$$
\widehat{\Omega}^{\mathcal{W}} \equiv \frac{\sum_{j=1}^{N} \sum_{k \neq j} \mathfrak{I}\left\{X_{j}\right\} \mathfrak{I}\left\{X_{k}\right\}}{\sum_{j=1}^{N} \sum_{k \neq j}\left|\mathfrak{I}\left\{X_{j}\right\} \mathfrak{I}\left\{X_{k}\right\}\right|}
$$

This estimator may also be written as a weighted statistic:

$$
\widehat{\Omega}^{\mathcal{W}}=\frac{\sum_{j=1}^{N} \sum_{k \neq j} W_{j, k} d\left(X_{j}, X_{k}\right)}{N(N-1) \bar{W}}
$$

where the weight $W_{j, k} \equiv\left|\mathfrak{I}\left\{X_{j} \mathfrak{I}\left\{X_{k}\right\}\right\}\right|, \bar{W}$ is the average weight, called weight normalization, defined as $\bar{W} \equiv \frac{1}{N(N-1)} \sum_{j=1}^{N} \sum_{k=j+1} W_{j, k}$, and the function $d$ is defined as:

$$
d(U, V) \equiv \operatorname{sgn}(\mathfrak{I}\{U\}) \operatorname{sgn}(\mathfrak{I}\{V\})
$$

where $U$ and $V$ are complex-valued random variables. Normalized weight is defined by the expression $W_{j, k} /(N(N-1) \bar{W})$ and sums up to one. Thus, dwPLI is obtained by computing the imaginary component of the crossspectra, computing the average imaginary component of the cross-spectra, and normalizing by the computed average over the magnitudes of the imaginary components of cross-spectra. If all weights $W_{j, k}$ equal 1 , then this equals the unbiased PLI-square estimator. dwPLI, wPLI, PLI, and other metrics were compared in LFP data recorded from the orbitofrontal cortex of the monkey (Van Wingerden et al., 2010).

\section{Band Limited Power Correlation}

A band limited power or amplitude envelop correlation provides functional coupling without coherence or phase coherence (Bruns et al., 2000). One typical approach for computing band limited amplitude or power envelope uses a Hilbert transform. Once the time series for each voxel in the source space is obtained by a source estimation of recorded magnetic fields, its analytic signal is then expressed using the Hilbert transform of the time series. The envelope of oscillatory activity is obtained by measuring the magnitude of the analytic signal, i.e., Hilbert envelopes. Pearson's correlation between Hilbert envelopes at different voxels is then computed as a metric of functional connectivity. This kind of correlation has been applied to ECoG signals (Bruns et al., 2000; Nir et al., 2008), MEG signals from normal and blind subjects (Brookes et al., 2011; Schepers et al., 2012), and LFP signals in monkeys (Leopold et al., 2003) and humans (Nir et al., 2008). Several studies have employed ICA to determine what signals need to be used in order to compute the connectivity of band-limited power time series. ICA itself is a linear family of blind source separation methods; however, it is often applied in MEG studies to nonlinear, band-limited power time series (Brookes et al., 2011; Luckhoo et al., 2012; Hall et al., 2013). Accordingly, this may also be regarded as a non-linear approach (Brookes et al., 2014). It has also been reported that changes in oscillatory power in MEG/EEG are associated with AMRI BOLD responses (Scheeringa et al., 2011). A recent study demonstrated that an fMRI BOLD correlation reflected a frequency-specific neuronal correlation of source-space signals in MEG data (Hipp and Siegel, 2015). Accordingly, the examination of power correlations is important when considering the relationship between the neuronal mechanisms underlying recorded MEG signals and those of BOLD signals.

\section{Power Envelope Correlation of Orthogonalized Signals}

Signal components that reflect the same source at two different sensors (or source estimates) are characterized by an identical phase. In contrast, for many cases, signals from different neuronal populations may be considered to have a variable phase relationship. This difference is used to remove the spurious zero-lag correlation of MEG signals caused by field spread in the sensor-space and signal leakage in the sourcespace (Hipp et al., 2012). In order to compute this, the orthogonalization of raw signals, i.e., removing the linear regression, is initially performed, which corresponds to the removal of signal components sharing the same phase, for each pair of signals, time window and carrier frequency. After orthogonalization, power envelopes are computed, and the linear correlation between these power envelopes is then computed as a measure of connectivity. This computation ensures that the signals do not share a trivial correlation in power resulting from methodological issues. Furthermore, a relevant technique, a residual envelope correlation, which has been extended from residual coherence, was recently suggested (Sekihara and Nagarajan, 2013).

\section{Mutual Information}

Mutual information (MI) is an information-theoretic metric of the mutual dependence of two random variables, and may be regarded as a decrease in uncertainty regarding one variable when knowledge of another is given. MI is based on Shannon entropy, which is a measure quantifying the uncertainty of a probability distribution. Shannon entropies of the marginal 
distributions of the systems $X$ and $Y$, and of the joint distribution are defined as:

$$
\begin{gathered}
H(X)=-\sum_{i=1}^{M_{x}} p_{x(i)} \log p_{x}(i) \\
H(Y)=-\sum_{i=1}^{M_{y}} p_{y(i)} \log p_{y}(i) \\
H(X, Y)=-\sum_{i=1}^{M_{x}} \sum_{j=1}^{M_{y}} p_{x y(i, j)} \log p_{x y}(i, j)
\end{gathered}
$$

MI is then defined as:

$$
I(X, Y)=H(X)+H(Y)-H(X, Y)
$$

MI is also interpreted as Kullback-Leibler entropy by measuring the gain the information when replacing the distribution of $p_{x}(i) p_{y}(j)$ by the actual joint distribution $p_{x y}(i, j)$, as follows:

$$
I(X, Y)=-\sum_{i=1}^{M_{x}} \sum_{j=1}^{M_{y}} p_{x y(i, j)} \log \frac{p_{x y}(i, j)}{p_{x}(i) p_{y}(j)}
$$

The base of the logarithm determines the units in which information is measured. A common example is to take base two, which means information to be measured in bits. The classical and common strategy to estimate MI from two time series, $x$ and $y$ involves partitioning the supports of $X$ and $Y$ into bins of finite size and counting the numbers of points falling into the various bins. MI has been successfully applied to MEG signals for speech processing (Cogan and Poeppel, 2011), cortical processing in the blind (Ioannides et al., 2013), or those with developmental disorders (Gómez et al., 2014), a graph-theory analysis of resting-state data (Jin et al., 2011, 2014), and musical expertise (Paraskevopoulos et al., 2015), as well as to EEG signals. In most of the above studies, the classical strategy was used to estimate MI, while other techniques (Kraskov et al., 2004) and correction techniques are available to overcome the binning bias issue (Roulston, 1999; Zhu et al., 2014).

\section{Comparisons of Functional Connectivity Metrics}

The metrics of functional connectivity described above differ from each other in sensitivity to a bias by common signal sources, linearity or non-linearity, and the included phase and amplitude information. iCoh, the PLI family, and power envelope correlation are less sensitive to spurious zero-lag connectivity caused by common cortical sources, including volume conduction, field spread, reference electrodes, and source leakage, and, thus, reflect true functional connectivity. Correlations, coherence, phase coherence, and PLV may provide spurious zero-lag connectivity between different cortical regions.
However, there are several conditions to which the latter metrics are applicable for examining functional connectivity; e.g., cortico-muscular connectivity (Gross et al., 2001, 2005), corticokinematic connectivity (Bourguignon et al., 2013; Marty et al., 2015), and spino-muscle connectivity (Takei and Seki, 2008). However, iCoh, the PLI family, and power envelope correlation may miss linear, but functionally meaningful interactions, which, in principle, may be expressed in zero or near-zero lag coherence. In spite of this potential omission, these metrics are free of any artifact of volume conduction, and do not cause type 1 errors (false positives). In contrast, other metrics such as correlations, coherence, and PLV may cause type 1 errors.

\section{EFFECTIVE CONNECTIVITY}

The functional connectivity metrics described above provide no directed flow of information or causality, whereas various methods have been suggested to infer directed information. We briefly summarize these below.

\section{Granger Causality Index}

Granger causality is a popular statistical concept of causality, which has been applied to several types of neuroscientific data in humans, non-human primates, and rodents. Interactions between two stochastic processes (or two signals) $X$ and $Y$ with the discrete time series $x(t)$ and $y(t)$ may be described by bivariate linear autoregressive (ARX) models:

$$
\begin{aligned}
& x(t)=\sum_{k=1}^{q} a_{11, k} x(t-k)+\sum_{k=1}^{p} a_{12, k} y(t-k)+e_{x}(t) \\
& y(t)=\sum_{k=1}^{q} a_{21, k} x(t-k)+\sum_{k=1}^{p} a_{22, k} y(t-k)+e_{y}(t)
\end{aligned}
$$

where $p$ and $q$ are the model orders of $y$ and $x$ regressors, $e_{x}(t)$ and $e_{y}(t)$ are the model prediction errors (residuals), and each is assumed to be serially uncorrelated over time. If the variance of $e_{x}(t)$ is reduced by adding the $Y$ terms in the first equation, $Y$ Granger-causes $X$, and vice versa. In other words, $Y$ Grangercauses $X$ if the coefficients in $a_{12}$ are jointly significantly different from zero. This may be examined by performing an $F$-test of the null hypothesis that $a_{12}=0$, given assumptions of covariance stationarity on $X$ and $Y$. The degree of a Granger causality interaction is estimated by the logarithm of the corresponding F-statistic. The Akaike Information Criterion (AIC) or Bayesian Information Criterion (BIC) is used to determine the appropriate model order.

\section{Dynamic Causal Modeling}

Dynamic causal modeling (DCM) was initially introduced in an fMRI study (Friston et al., 2003). DCM is a metric of causality, and enables us to examine how brain areas within a network interact. It is a generic Bayesian framework for inferring hidden neuronal states from measurements of hemodynamic or electromagnetic activity in the brain. DCM models the cognitive 
system at its underlying neuronal level (called the neuronal mass model). The modeled neuronal dynamics are transformed into area-specific BOLD signals by a hemodynamic model. The aim of DCM is to estimate parameters at the neuronal level such that the modeled and measured BOLD signals are optimally similar. The first introduction of DCM was a bilinear version (Friston et al., 2003), and a non-linear version is also now available (Stephan et al., 2008). DCM is also available for MEG/EEG signals (FitzGerald et al., 2015; Garrido et al., 2015; Klingner et al., 2015). Studies utilizing DCM have investigated a wide range of topics; motor network (Volz et al., 2015), action observation (Thioux and Keysers, 2015), sense of agency (Ritterband-Rosenbaum et al., 2014), attention (Brown and Friston, 2013), handedness (Pool et al., 2014), cognitive control and working memory (Harding et al., 2015), naturalistic communication (Regenbogen et al., 2013), neurodevelopmental disorders (Albouy et al., 2015), steady-state visual evoked responses with simultaneous EEG-fMRI recordings (Youssofzadeh et al., 2015), and applications to ECoG signals in the monkey (Bastos et al., 2015).

\section{Causality Metrics in the Frequency Domain}

As discussed above, although Granger causality is a statistic of causality computed in the time domain, relevant statistics in the frequency domain may also be calculated, including directed transfer function (DTF) (Kaminski and Blinowska, 1991), partial directed coherence (PDC) (Baccalá and Sameshima, 2001), and spectral Granger causality (Geweke, 1982) metrics. A non-linear PDC was recently suggested and applied to EEG signals from an epileptic patient (He et al., 2014).

\section{Transfer Entropy}

Transfer entropy is an information-theoretic measure of timedirected information transfer between two random processes (Schreiber, 2000). TE measures the rate of information flow between two variables as a violation of the Markov property, which is a memoryless property of a stochastic process. TE from two time series $x_{t}$ and $y_{t}$ is written as:

$$
\begin{aligned}
& \operatorname{TE}(X \rightarrow Y) \\
& =\sum_{y_{t}+u, y_{t}^{d_{y}}, x_{t}^{d_{x}}} p\left(y_{t+u}, \boldsymbol{y}_{t}^{d_{y}}, \boldsymbol{x}_{t}^{d_{x}}\right) \log \frac{p\left(\left(y_{t+u} \mid \boldsymbol{y}_{t}^{d y} \boldsymbol{x}_{t}^{d x}\right)\right)}{p\left(y_{t+u} \mid \boldsymbol{y}_{t}^{d_{y}}\right)}
\end{aligned}
$$

where $t$ is a time index, $u$ is the prediction time, and $y_{t}^{d_{y}}$ and $x_{t}^{d_{x}}$ are $d_{x^{-}}$and $d_{y}$-dimensional delay vectors. In contrast to Granger causality relating to prediction, TE is framed in terms of the resolution of uncertainty. The Granger Causality family described above is also a data-driven, model-based metric of causality and DCM is a biologically inspired, model-based approach, whereas TE is a model -free method. Thus, TE is advantageous for exploratory analyses over the Granger causality and other model-based methods, especially for the detection of unknown non-linear interactions. TE fulfills several important requirements for detecting causal relationships between two time series in neuroscience (Vicente et al., 2011). It does not require the a priori definition of the type of interaction. It may detect frequently observed types of purely non-linear interactions. Furthermore, TE detects causal relationships even when a wide distribution of delays exists in interactions between two time series. TE is also robust against linear cross-talk between time series. TE has been applied to MEG and EEG studies on auditory speech (Park et al., 2015), simple motor tasks (Vicente et al., 2011), auditory working memory tasks (Wibral et al., 2011), and an epileptic patient (Chávez et al., 2003). Early fMRI, EEG, and MEG studies (Hinrichs et al., 2006, 2008) used a very similar measure, called directed information transfer (DIT), on the basis of directed transinformation (Saito and Harashima, 1981; Kamitake et al., 1984); however, this computation appears to limit the detection of directed interactions to linear ones (Vicente et al., 2011). In addition, a multivariate extension of transfer entropy and MI (Lizier et al., 2011) and a phase version of TE have been proposed (Lobier et al., 2014).

\section{Phase Slope Index}

The phase slope index (PSI) is a metric used to estimate the direction of information flux during multivariate time series from complex systems and was proposed by Nolte and colleagues (Nolte et al., 2008b). This metric is based on the slope of the phase spectrum, and has invariance properties that are important for applications in real physical or biological systems. PSI is strictly insensitive to mixtures of arbitrary independent sources, gives meaningful results even if the phase spectrum is not linear, and properly weighs contributions from different frequencies. Compared with standard Granger causality, PSI has the ability to detect true interactions, even in mixed and noisy complex systems. In the Causality Challenge, PSI provided a higher score $(+578)$ than Granger causality (-264) (Nolte et al., 2008a). It has also been applied to source-space EEG data (Nolte and Müller, 2010).

\section{APPROPRIATE METRICS OF CONNECTIVITY}

The question of what metric is preferable for representing interregional relationships is ultimately a statistical issue, and also depends on the research of interest. This choice will depend on which types of errors, false positives and negatives, are acceptable for the respective research and whether researchers take the risk of making either error. Another point of view is associated with the specific research question about interdependence between signals, regions, or voxels, namely, what type of interdependence between two signals are researchers interested in: correlation, phase, or directed influence. This means that researchers need a clear scientific hypothesis and the resulting experimental hypotheses that determine what type of metrics should be used. It is important to consider these differences including advantages and disadvantages and to employ appropriate measures for individual research. An opposite point of view on the selection of metrics to be used is also important. For example, researchers must choose an arbitrary one of connectivity metrics on the basis of ambiguous information in an exploratory study that makes it difficult to determine the metric to be used prior to the experiment. Although this is an inefficient approach, it does exist. 
This is important for effective connectivity metrics because some of these need the a priori definition of the type of interaction to be computed; however, researchers often cannot obtain a priori information, especially in a large-scale data set. TE or other variants may be more appropriate in such exploratory cases of effective connectivity.

\section{VARIOUS SOURCE ESTIMATION ALGORITHMS USED IN CONNECTIVITY STUDIES}

Various source estimation algorithms are currently available, as described partly in the Basics of MEG/EEG section; however, computations of connectivity from MEG/EEG data require timeseries data or converted frequency-series data. Accordingly, source estimation algorithms to preprocess the computation of source-space connectivity may fulfill some requirements; e.g., (1) the reconstruction of time-series data in the source space is possible, (2) the number of estimated sources in addition to the location and orientation of each source are accurate, and (3) computation demand is as low as possible. Some algorithms themselves provide source-space connectivity, e.g., the DICS algorithm reported by Gross and colleagues regarding coherence, as described in the earlier Coherence section. According to Gross et al. (2010), at least three strategies are available for identifying significant connectivity pairs in the source space (Gross et al., 2010); (1) the selection of individual anatomical brain regions based on a priori information such as findings from other studies and subsequent tests for the connectivity of time-series data between selected regions, (2) the selection of regions based on their activity and subsequent tests for connectivity, and (3) the selection of regions directly based on their connectivity. Most of the available source reconstruction algorithms belong to the second category, whereas DICS belongs to the third category. The opposite strategy for connectivity studies is the brain-wide computation of connectivity in the source space, i.e., computations of connectivity for all pairs of available voxels (or meshes, virtual sensors, and dipoles).

As distributed source estimation algorithms, there are minimum-norm techniques, magnetic field tomography (MFT), and beamforming available for subsequent computations of connectivity. Regarding minimum-norm techniques, one previous study filtered MEG signals with a Morlet wavelet and then reconstructed sources on a single-trial basis with dynamic statistical parametric mapping (dSPM) (Lin et al., 2004). This study showed power estimates normalized by the baseline value on a cortical surface without selecting regions of interest, and PLV was also mapped as an index of phase synchronization. David and colleagues detected active voxels using a similar source reconstruction algorithm, but with surrogate data. Nonaveraged data were used for source localization, and the iteration procedure employed reduced the number of active voxels, which provided the sparse and focal source representations used for computations of connectivity (David et al., 2002, 2003). Minimum-norm source reconstruction was also applied to subsequent computations of the frequency flow metric, which is based on an estimation of the instantaneous frequency in the time-frequency plane (Amorim et al., 2005; Rudrauf et al., 2006). In another study, the DTF (Kaminski and Blinowska, 1991) was also computed as an index of effective connectivity after MNE (Astolfi et al., 2005). Synchronization tomography is another class consisting of a source estimation algorithm and subsequent computation of phase synchronization (Tass et al., 2003). Source estimation is based on MFT, a non-linear localization algorithm, to compute current densities with the brain for each recorded data sample (Ioannides et al., 1990; Poghosyan and Ioannides, 2008). MFT may be applied to the non-averaged data used to compute subsequent MI and time-delayed MI as an index of functional connectivity (Ioannides et al., 2000, 2004, 2013).

Other algorithms are based on the ECD estimation with relatively small numbers of ECDs. These consist of computations of source coherence using a multidipole model (Hoechstetter et al., 2004) and phase synchronization of independent components (ICs) separated from recorded data using an independent component analysis (ICA) implemented in EEGLAB (Makeig et al., 2004a,b). A recent source estimation algorithm, called Champagne, uses a novel empirical Bayesian scheme to estimate the number and location of a small (sparse) set of flexible dipoles that adequately account for the observed sensor-space data (Owen et al., 2009, 2012; Wipf et al., 2010). These studies compared the abilities of Champagne, MCE, beamforming, and sLORETA/dSPM to detect connectivity with coherence, iCoh, and multivariate autoregression (MVAR). MVAR is a valuable tool because it quantifies the time scale of interactions and provides directionality by modeling data as the linear mixing of current and past values of the data. An empirical analysis with various datasets showed that Champagne outperformed other estimation algorithms in terms of localization accuracy and time-course reconstruction at various signal-to-noise ratios. Therefore, the high suitability of this algorithm for functional connectivity analyses was suggested because it is robust to highly correlated dipoles and circumvents the issue of computational complexity by vastly pruning the number of active voxels (only 4-7 dipoles were estimated and used to compute connectivity metrics). Consequently, Champagne also functions to reduce the spatial dimension of data on the basis of the MEG signal itself, which is reasonable for computing functional connectivity in terms of decreasing computational demand and avoiding source leakage. Thus, sparse source configurations including Champagne are very useful for examining connectivity. In contrast, connectivity may need to be computed for all pairs of as many dipoles as possible (more than one thousand) in order to prevent any sign of small interactions being missed, and also to examine graph metrics computed from the brain-wide connectivity matrix. Both approaches are highly valuable from a scientific point of view, and, thus, differentiating between them is also considered important. Champagne is well suitable for source-space functional connectivity under various experimental conditions, in which only a small number of activated regions are predicted, and may be applied to an examination of instantaneous connectivity. In contrast, brain-wide computations of source-space connectivity and a subsequent network analysis requires source reconstructions 
by beamforming or MCE, MNE naturally, and the use of noninstantaneous connectivity metrics in order to avoid spurious connectivity between local regions.

\section{EEG-TMS TECHNIQUE FOR EFFECTIVE CONNECTIVITY}

Another technique to examine effective connectivity involves the integrative use of recordings of brain activity and stimulation of the brain region. A typical example is the EEG-TMS technique (Ilmoniemi et al., 1997). By recording EEG responses to a transcranial stimulation of the brain region, the EEG-TMS technique allows us to estimate the time course of the causal effects of the stimulated regions on other regions in which the recorded responses may originate. This technique has been applied to studies on attention (Morishima et al., 2009), task and decision inertia (Akaishi et al., 2010, 2014), and perceptual decision making (Akaishi et al., 2013). Similar techniques are also possible in other recording modalities, such as PET-TMS (Paus et al., 1997), fMRI-TMS (Bestmann et al., 2004), and NIRS-TMS (Noguchi et al., 2003). These techniques are based on artificial neuronal impulses evoked by TMS, but are also applicable to examinations of the actual involvement of the stimulated and recorded regions by integrating with the virtual lesion technique. In the near future, these techniques may be integrated with connectivity metrics, which were reviewed above, and/or graph metrics, which are reviewed below. This integration may open a new avenue for examining the dynamics of activity magnitude, connectivity, network, and the actual involvement of brain regions. The invasive type of this technique is also available (Matsumoto et al., 2012). A comprehensive consensus paper (Siebner et al., 2009) and Research Topic in Frontiers ("Manipulative approaches to human brain dynamics" edited by Kitajo, Hanakawa, Ilmoniemi, and Miniussi) are helpful for learning more about these integrative techniques.

\section{NETWORK OR GRAPH METRICS DERIVED FROM A GRAPH-THEORETICAL ANALYSIS}

In previous sections, we discussed various functional and effective connectivity metrics computed from functional brain signals, which basically describe various statistical relationships between two signals and are a collection or averaged representation of only a very small fraction of a network. The third category (network or graph) provides higherlevel perspectives of functional organization in the human brain network. A graph-theoretical analysis computes metrics describing the various local and global properties of a network, and takes many connectivity values into consideration. This powerful analysis of neuroimaging data has revealed interesting network properties including small-world organization, scalefree features, hubs, modularity, and rich clubs. A typical method used to obtain graph metrics is to initially compute connectivity values between two signals for all pairs of nodes, and then perform a graph-theoretical analysis on the connectivity matrix obtained. In an unweighted graph, connectivity values are thresholded with certain criteria and binarized before the computation of graph metrics, while weighted graph metrics are computed from an original connectivity matrix without thresholding. This analysis has been applied to structural and functional MRI data, and detected the structural core in the posterior medial and parietal regions, which markedly overlapped with the default-mode network (Hagmann et al., 2008). Its application to macaque data has also been reported (Honey and Sporns, 2008). This map of neural connections within the brain is now regarded as a connectome (Hagmann, 2005; Sporns et al., 2005). The first application of the graph theory to fMRI data demonstrated various clusters in the form of subgraphs in a finger tapping task (Dodel et al., 2002). Stam (2004) first performed a graph-theoretical analysis on resting-state MEG datasets in the sensor-space (Stam, 2004), and subsequently on resting-state EEG datasets (Stam et al., 2007a). Bassett and colleagues also reported a small-world architecture together with the fractal organization using sensor-space MEG data (Bassett et al., 2006). This has provided an abundant amount of evidence to support alterations in network properties in various neurological diseases (Stam, 2014). We herein briefly summarize relevant important graph metrics. Please refer to recent review articles for more details (Bullmore and Sporns, 2009; Rubinov and Sporns, 2010; Sporns, 2013; Sporns and Bullmore, 2014; Stam, 2014).

\section{Degree}

The degree of the vertex of a graph is the number of edges incident to the vertex, with loops counted twice. Thus, individual values of the degree characterize the importance of vertices in the network. The degree distribution is the probability distribution of these degrees over the whole network, and an important metric of network development and resilience. The mean network degree is commonly used as a metric of density, or the total wiring cost of the network (Rubinov and Sporns, 2010). In a directed graph, nodes have two different degrees, the in-degree and outdegree, which are the numbers of incoming and outgoing edges, respectively. In a weighted graph, instead of the degree, the same type of metric is called the strength, which equals the sum of all neighboring edge weights.

\section{Clustering Coefficient and Transitivity}

A clustering coefficient is a metric of the degree to which vertices in a graph cluster together. There are two modes of clustering coefficients; local and global. The local clustering coefficient of a vertex is the proportion of edges between vertices within its neighborhood divided by the number of edges that may exist between them (Watts and Strogatz, 1998). Accordingly, the local clustering coefficient is 1 if the neighborhood is fully connected, whereas the value is 0 if there are no connections in the neighborhood. Therefore, this value represents how well connected the neighborhood of the node is ("cliquishness"). The average of local clustering coefficients across all vertices in a graph, often called the mean clustering coefficient, reflects the overall level of clustering in a network (Watts and Strogatz, 1998). This means the clustering coefficient is also regarded as an alternative to the global clustering coefficient (also called 
transitivity), which is a classical metric to measure the overall level of clustering (Luce and Perry, 1949; Wasserman and Faust, 1994). The local clustering coefficient is one of the factors determining the small-world feature of a network by Watts and Strogatz, while another is the characteristic path length. The ratio of the local clustering coefficient and weighted averaged shortest path length is called small-worldness, and has been applied to MEG signals (Stam et al., 2009; Douw et al., 2011).

\section{Characteristic Path Length}

The characteristic path length is the average shortest path length between all pairs of nodes in a network (Watts and Strogatz, 1998). As the name suggests, the shortest path is defined as the shortest path from one vertex to another in a network, which is computed as the least number of or least total weight of edges. Various algorithms have been suggested to compute the shortest path, including Dijkstra's algorithm, its modifications, the Bellman-Ford algorithm, Gabow's algorithm, the Floyd-Warshall algorithm, Pettie-Ramachandran algorithm, and Johnson algorithm.

\section{Local Efficiency}

The efficiency of a network is a metric of the efficiency of information exchange in network science. Local and global types of efficiency have been identified. Local efficiency is computed as the inverse of the shortest path length between connected vertices that are neighbors with the vertex of interest (Latora and Marchiori, 2001). Therefore, local efficiency quantifies how well information is exchanged by its neighbors when it is removed, and, thus, is a metric of the resistance of a network to failure at the local level. This is also regarded as an alternative to the local clustering coefficient.

\section{Global Efficiency}

Global efficiency is computed by dividing the average efficiency of a graph with that of an ideal graph, which is comparable to the inverse of the average path length of a network (Latora and Marchiori, 2001). However, the inverse of the average path length measures efficiency in a system in which one packet information is being moved via the network, whereas global efficiency measures efficiency when all the vertices are exchanging packets of information with each other. Global efficiency was previously shown to be easier to use numerically than the path length (Bullmore and Sporns, 2009). Local and global efficiency metrics are valuable when examining physical biological networks.

\section{Modularity}

Modularity is a metric that describes the structure of a network, and is computed as the fraction of the edges that fall within the given groups minus the expected fraction if edges were randomly distributed. Thus, modularity represents the degree to which a network is subdivided into non-overlapping groups (also called modules, clusters, and communities). Computation techniques for modularity utilize optimization algorithms (Newman, 2006; Blondel et al., 2008; Leicht and Newman, 2008). Other algorithms also detect overlapping community structures (Palla et al., 2005).

\section{Degree Centrality}

Important brain regions, sometimes called hubs or cores, interact with other regions, and may be the center of information flow. This may be expressed by how many individual vertices connect with others or how many of the shortest paths the vertex is involved in. Degree centrality is one of the most common metrics of centrality. Vertices with a high degree of centrality connect with more vertices than those with a low degree of centrality.

\section{Closeness Centrality and Betweenness Centrality}

Closeness centrality represents how close individual vertices are to others on average in a network, and is computed as the inverse of the average shortest path length from a vertex to all other vertices in the network (Sabidussi, 1966; Freeman, 1978). Betweenness centrality is a metric of the importance of the vertex in a network (Freeman, 1978). This metric is a computationally expense among centrality metrics but is very popular. A large number of neuroimaging studies have successfully used betweenness centrality to reflect the importance of a network (Hipp et al., 2012; Kida and Kakigi, 2013; Kida et al., 2013) and degree distribution. Computations of betweenness centrality and closeness centrality are both based on the computation of short paths. Several algorithms including Brandes's (Brandes, 2001) and Kintali's algorithms (Kintali, 2008) have been proposed in order to compute betweenness centrality. Closeness and betweenness centralities are based on central vertices being involved in many of the shortest paths in a network, and, consequently, function as important controls of information flow (Freeman, 1978; Rubinov and Sporns, 2010).

\section{Motifs}

A motif is a generalized version of the clustering coefficient, and a pattern of local connectivity. The presence of a motif is demonstrated by showing the frequent occurrence of that pattern in the network, and is actually examined with the motif z-score compared with the surrogate data (Milo et al., 2002).

\section{Small-Worldness}

The clustering coefficient and averaged shortest path length are metrics representing the small-world feature of a network. A second-order graph statistic, which is a metric of smallworldness, is computed by dividing the ratio of the clustering coefficient in relation to surrogate data by that of the averaged shortest path length in relation to surrogate data (Humphries and Gurney, 2008). This metric has been used in sensor-space MEG data (Douw et al., 2011).

\section{BRAIN-WIDE ANALYSIS OF CONNECTIVITY AND GRAPH METRICS}

In recent years, MEG studies have conducted a brain-wide source-space analysis of resting-state functional connectivity (De Pasquale et al., 2010; Brookes et al., 2011; Hipp et al., 2012) and graph metrics (Hipp et al., 2012). Since connectivity is as transient, adaptive, and context-sensitive as brain activity 
per se (Friston, 2011), another important issue in MEG/EEG studies is rapid changes in graph metrics during task states in the source-space. Bassett and colleagues reported task-related changes in the degree and betweenness centrality of the gammaband network in the sensor space with the execution of visuallyinitiated continuous finger tapping at $1.2 \mathrm{~Hz}$ for approximately $10 \mathrm{~s}$ (4 repetitions for each participant) compared to a resting condition. This does not measure rapid changes in graph metrics, while recent studies reported brain-wide, task-related temporal changes in graph metrics computed from full sensor-space data in a multisensory attention task (Kida and Kakigi, 2013) and working memory task (Ariza et al., 2015). A recent EEG study reported brain-wide network properties computed from source-space data in a visual discrimination task (Bola and Sabel, 2015). However, most EEG/MEG studies performing the computation of graph metrics in the source-space have divided the brain into approximately 100 or fewer regions (voxels or grid points, meshes) based on various brain atlases (such as AAL, Talairach, and Harvard-Oxford) in order to compute source activity, connectivity, and graph metrics. In contrast, the first demonstration of the human structural connectome using a graph-theory analysis by Hagmann and colleagues was provided based on a computation with 998 vertices (Hagmann et al., 2008). The brain-wide graph-theory analysis of resting-state MEG by Hipp and colleagues was performed with 2926 vertices in the source space (Hipp et al., 2012). Therefore, larger numbers of vertices are preferable for computing the brain-wide analysis of task-related, rapid changes in graph metrics in MEG and EEG studies. A preliminary MEG study reported task-related, frequency-specific, rapid temporal changes in the brain-wide network organization by examining graph metrics computed from full source-space data (about 900 vertices) in a multisensory attention task (Kida et al., 2013). The findings of this study indicated the possibility of the cortical dynamics of functional networks showing frequency-specific, sub-second rapid temporal changes depending on the periods of task execution. fMRI studies have also been investigating brain-wide task-related temporal changes in graph metrics (Wang et al., 2013). This type of exhaustive analysis handling a full data space, which includes several categories of neural indices in space, time, and frequency dimensions, is an extremely time-consuming task with personal computers or workstations and may feasibly be achieved using parallel computing or GPU computing, besides supercomputers. In contrast, dimensional reductions in source space data such as a minimum spanning tree or clustering makes the computations of graph metrics efficient. Thus, advances in this line of research will undoubtedly lead to more exciting discoveries on brain mechanisms based on the activity strength, connectivity, and network along different dimensions (space, time, and frequency).

\section{COMPLEX DYNAMICS OF THE HUMAN BRAIN}

Brain signals with high-temporal resolution such as MEG are feasible for examining dynamical systems in the brain, which works rapidly and adaptively. Many events in the real world show complex behaviors that cannot simply be explained by linear equations, whereas others can. Complex systems is a collective term to describe a system showing various properties and functions such as fractal (self-similarity), chaos, cellular automaton, percolation, and self-organized criticality, by largescale interactions or strong non-linearity between elements involved in the system. Complex systems have a wide variety of real-world examples including social organization, artificial intelligence, ecosystems, earthquakes, living cells, the universe and, of course, the brain. The identification of complex systems in the human brain has been associated with connectivity and network analyses, and, thus, we briefly review MEG/EEG studies characterizing complex dynamics in the human brain.

\section{Fractal Scaling}

Fractals are a measure of self-similarity, which is an important concept characterizing complex dynamics. fMRI studies performed the fractal scaling of low frequency human brain activation, mainly in a resting state (Bullmore et al., 2004; Maxim et al., 2005; Achard et al., 2008; Suckling et al., 2008; Wink et al., 2008). Classical studies reported the feasibility of a fractal analysis of stereo-EEG (SEEG) signals directly recorded from the cortex of epileptic patients for a diagnosis (Bullmore et al., 1994). Bassett and colleagues used MEG to demonstrate that human brain oscillations showed scale-invariant or fractal scaling over the frequency range of $1-75 \mathrm{~Hz}$ (Bassett et al., 2006). MEG signals recorded using a 275-ch whole head system (axial type) in a resting state and during finger tapping, and computed graph metrics (average path length, clustering, sigma, characteristic path length), synchronizability, and dynamic parameters (such as the power law exponent and exponential cut-off degree). All these metrics showed scale invariance or fractal scaling over the frequency range. However, major changes were not observed in global network parameters between visually-cued oscillatory finger tapping and the resting condition; however, minor changes were noted in the degree and betweenness centrality in premotor and prefrontal regions in the gamma-band network. Thus, a fractal analysis of MEG/EEG signals as well as fMRI provides valuable insights into self-similarity involved in the complex systems of the human brain, and the sub-second dynamics of the fractal dimension following task execution warrant further studies.

\section{Self-Organized Criticality or the Neuronal Avalanche}

Self-organized criticality is an interesting model in complex systems (Bak et al., 1987). In their early study, Bak and colleagues indicated that systems consisting of many elements with nonlinear interactions self-organized into a state with statistical signs of critical systems, with spatial and temporal correlations of a power-law form being the statistical signs. The power-rule form is also called "scale-free" because it is the only mathematical function that indicates that the system has no typical or characteristic scale. The branching ratio is an important metric of criticality. There is a large amount of evidence to support selforganized criticality in non-human animal neurophysiology in vitro in terms of the branching ratio. Previous studies reported 
that the LFPs of spontaneous activity showed spatiotemporal cascades of discrete events, called the neuronal avalanche, in acute cortex slices, slice cultures, and the developing cortex of the anesthetized rat (Beggs and Plenz, 2003; Gireesh and Plenz, 2008; Lombardi et al., 2012; Yang et al., 2012; Shew and Plenz, 2013). This neuronal avalanche has also recently been detected in spontaneous LFPs and spike activity in vivo in sensory and motor-related regions (Petermann et al., 2009; Hahn et al., 2010). These cascades are characterized by a critical branching process. In branching processes, activity propagates from one active group of neurons to another in a cascade. Here, the ratio between activations in consecutive time steps is termed the branching ratio, $\sigma$. If the branching ratio is smaller than one, activity dies out before it has propagated far (sub-critical network), whereas a ratio larger than one produces an explosion of activity (super-critical network). When the ratio is around one, the system is critical and the activation of $N$ neurons leads to the activation of other $N$ neurons, operating at an exquisite balance at which activity propagates long distances without runaway excitation of the super-critical network. Various kinds of analyses of resting-state MEG signals have provided evidence for the presence of self-organized criticality by examining longrange temporal correlations and power-law scaling behavior (Linkenkaer-Hansen et al., 2001), the lifetime of the oscillatory avalanche (Poil et al., 2008), synchrony-based power-law scaling (Kitzbichler et al., 2009), and the size of the avalanche cascade (Shriki et al., 2013). Recent MEG and EEG studies reported a correlation between a long-range temporal correlation or avalanche dynamics and behavioral scaling laws (Palva et al., 2013; Smit et al., 2013) and developmental changes (Smit et al., 2011). Thus, the neuronal avalanche as a notable factor for

\section{REFERENCES}

Achard, S., Bassett, D. S., Meyer-Lindenberg, A., and Bullmore, E. (2008). Fractal connectivity of long-memory networks. Phys. Rev. E Stat. Nonlin. Soft Matter Phys. 77:036104. doi: 10.1103/PhysRevE.77.036104

Adey, W. R., Walter, D. O., and Hendrix, C. E. (1961). Computer techniques in correlation and spectral analyses of cerebral slow waves during discriminative behavior. Exp. Neurol. 3, 501-524. doi: 10.1016/S0014-4886(61)80002-2

Aertsen, M. H., and Preissl, H. (1991). "Dynamics of activity and connectivity in physiological neuronal networks," in Nonlinear Dynamics and Neuronal Networks, eds H. G. Schuster (New York, NY: VCH Publishers), 281-301.

Ahlfors, S. P., Han, J., Belliveau, J. W., and Hämäläinen, M. S. (2010). Sensitivity of MEG and EEG to source orientation. Brain Topogr. 23, 227-232. doi: 10.1007/s10548-010-0154-x

Aine, C. J., Supek, S., George, J. S., Ranken, D., Lewine, J., Sanders, J., et al. (1996). Retinotopic organization of human visual cortex: departures from the classical model. Cereb. Cortex 6, 354-361. doi: 10.1093/cercor/6.3.354

Akaishi, R., Morishima, Y., Rajeswaren, V. P., Aoki, S., and Sakai, K. (2010). Stimulation of the frontal eye field reveals persistent effective connectivity after controlled behavior. J. Neurosci. 30, 4295-4305. doi: 10.1523/JNEUROSCI.6198-09.2010

Akaishi, R., Ueda, N., and Sakai, K. (2013). Task-related modulation of effective connectivity during perceptual decision making: dissociation between dorsal and ventral prefrontal cortex. Front. Hum. Neurosci. 7:365. doi: $10.3389 /$ fnhum.2013.00365

Akaishi, R., Umeda, K., Nagase, A., and Sakai, K. (2014). Autonomous mechanism of internal choice estimate underlies decision inertia. Neuron 81, 195-206. doi: 10.1016/j.neuron.2013.10.018 identifying cortical dynamics at criticality has recently been suggested.

\section{SUMMARY}

This review focused on connectivity and network metrics derived from MEG/EEG signals in space, time, and frequency dimensions. Efficient analyses of MEG and EEG signals in the full data space may uncover hidden information embedded in these signals. The fusion of non-linear physical science and neuroscientific MEG/EEG studies has been characterizing the dynamic and complex systems of the human brain, and self-organized criticality and self-similarity are representatives. Therefore, this review shows that these sophisticated analysis methods of MEG/EEG signals hold tremendous potential for uncovering the various functions of the human brain in multidimensional space.

\section{AUTHOR CONTRIBUTION}

TK, ET, and RK contributed to drafting and revising the paper.

\section{ACKNOWLEDGMENTS}

Our studies described in this review were supported by Grantsin-Aid for Young Scientists (A) (No. 25705021) and Exploratory Research (No. 25590211, No. 15K13163) from the Japan Society for the Promotion of Science (JSPS) to TK, and by COI STREAM (Center of Innovation Science and Technology based Radical Innovation and Entrepreneurship Program) from the Ministry of Education, Culture, Sports, Science and Technology, Japan.

Albouy, P., Mattout, J., Sanchez, G., Tillmann, B., and Caclin, A. (2015). Altered retrieval of melodic information in congenital amusia: insights from dynamic causal modeling of MEG data. Front. Hum. Neurosci. 9:20. doi: 10.3389 /fnhum. 2015.00020

Allison, T., McCarthy, G., Wood, C. C., and Jones, S. J. (1991). Potentials evoked in human and monkey cerebral cortex by stimulation of the median nerve. A review of scalp and intracranial recordings. Brain 114(Pt 6), 2465-2503. doi: 10.1093/brain/114.6.2465

Allison, T., Puce, A., Spencer, D. D., and McCarthy, G. (1999). Electrophysiological studies of human face perception. I: potentials generated in occipitotemporal cortex by face and non-face stimuli. Cereb. Cortex 9, 415-430. doi: 10.1093/cercor/9.5.415

Amorim, B. J., Etchebehere, E. C., Camargo, E. E., Rio, P. A., Bonilha, L., Rorden, C., et al. (2005). Statistical voxel-wise analysis of ictal SPECT reveals pattern of abnormal perfusion in patients with temporal lobe epilepsy. Arq. Neuropsiquiatr. 63, 977-983. doi: 10.1590/S0004-282X2005000600014

Andrew, C., and Pfurtscheller, G. (1996). Dependence of coherence measurements on EEG derivation type. Med. Biol. Eng. Comput. 34, 232-238. doi: 10.1007/BF02520079

Ariza, P., Solesio-Jofre, E., Martínez, J. H., Pineda-Pardo, J. A., Niso, G., Maestú, F., et al. (2015). Evaluating the effect of aging on interference resolution with time-varying complex networks analysis. Front. Hum. Neurosci. 9:255. doi: 10.3389/fnhum.2015.00255

Arnhold, J., Grassberger, P., Lehnertz, K., and Elger, C. E. (1999). A robust method for detecting interdependences: application to intracranially recorded EEG. Physica D 134, 419-430. doi: 10.1016/S0167-2789(99)00140-2

Astolfi, L., Cincotti, F., Mattia, D., Babiloni, C., Carducci, F., Basilisco, A., et al. (2005). Assessing cortical functional connectivity by linear inverse estimation 
and directed transfer function: simulations and application to real data. Clin. Neurophysiol. 116, 920-932. doi: 10.1016/j.clinph.2004.10.012

Babadi, B., Obregon-Henao, G., Lamus, C., Hämäläinen, M. S., Brown, E. N., and Purdon, P. L. (2014). A Subspace Pursuit-based Iterative Greedy Hierarchical solution to the neuromagnetic inverse problem. Neuroimage 87, 427-443. doi: 10.1016/j.neuroimage.2013.09.008

Babiloni, F., and Astolfi, L. (2014). Social neuroscience and hyperscanning techniques: past, present and future. Neurosci. Biobehav. Rev. 44, 76-93. doi: 10.1016/j.neubiorev.2012.07.006

Babiloni, F., Cincotti, F., Mattia, D., Mattiocco, M., De Vico Fallani, F., Tocci, A., et al. (2006). Hypermethods for EEG hyperscanning. Conf. Proc. IEEE Eng. Med. Biol. Soc. 1, 3666-3669. doi: 10.1109/iembs.2006.260754

Baccalá, L. A., and Sameshima, K. (2001). Partial directed coherence: a new concept in neural structure determination. Biol. Cybern. 84, 463-474. doi: 10.1007/PL00007990

Bak, P., Tang, C., and Wiesenfeld, K. (1987). Self-organized criticality: an explanation of the $1 / \mathrm{f}$ noise. Phys. Rev. Lett. 59, 381-384. doi: 10.1103/PhysRevLett.59.381

Bandettini, P. A., Wong, E. C., Hinks, R. S., Tikofsky, R. S., and Hyde, J. S. (1992). Time course EPI of human brain function during task activation. Magn. Reson. Med. 25, 390-397. doi: 10.1002/mrm.1910250220

Barabasi, A. L., and Albert, R. (1999). Emergence of scaling in random networks. Science 286, 509-512. doi: 10.1126/science.286.5439.509

Bartels, A., and Zeki, S. (2004). The neural correlates of maternal and romantic love. Neuroimage 21, 1155-1166. doi: 10.1016/j.neuroimage.2003.11.003

Bassett, D. S., Meyer-Lindenberg, A., Achard, S., Duke, T., and Bullmore, E. (2006). Adaptive reconfiguration of fractal small-world human brain functional networks. Proc. Natl. Acad. Sci. U.S.A. 103, 19518-19523. doi: $10.1073 /$ pnas. 0606005103

Bastos, A. M., Litvak, V., Moran, R., Bosman, C. A., Fries, P., and Friston, K. J. (2015). A DCM study of spectral asymmetries in feedforward and feedback connections between visual areas V1 and V4 in the monkey. Neuroimage 108, 460-475. doi: 10.1016/j.neuroimage.2014.12.081

Baumgartner, C., Doppelbauer, A., Deecke, L., Barth, D. S., Zeitlhofer, J., Lindinger, G., et al. (1991). Neuromagnetic investigation of somatotopy of human hand somatosensory cortex. Exp. Brain Res. 87, 641-648. doi: 10.1007/BF002 27089

Beauchamp, M. S., Lee, K. E., Argall, B. D., and Martin, A. (2004). Integration of auditory and visual information about objects in superior temporal sulcus. Neuron 41, 809-823. doi: 10.1016/S0896-6273(04)00070-4

Beggs, J. M., and Plenz, D. (2003). Neuronal avalanches in neocortical circuits. J. Neurosci. 23, 11167-11177.

Berger, H. (1929). Ueber das Elektrenkephalogramm des, Menschen. Archiv für Psychiatrie 87, 527-570. doi: 10.1007/BF01797193

Bestmann, S., Baudewig, J., Siebner, H. R., Rothwell, J. C., and Frahm, J. (2004). Functional MRI of the immediate impact of transcranial magnetic stimulation on cortical and subcortical motor circuits. Eur. J. Neurosci. 19, 1950-1962. doi: 10.1111/j.1460-9568.2004.03277.x

Blondel, V. D., Guillaume, J. L., Lambiotte, R., and Lefebvre, E. (2008). Fast unfolding of communities in large networks. J. Stat. Mech. 2008:P10008. doi: $10.1088 / 1742-5468 / 2008 / 10 /$ P10008

Bola, M., and Sabel, B. A. (2015). Dynamic reorganization of brain functional networks during cognition. Neuroimage 114, 398-413. doi: 10.1016/j.neuroimage.2015.03.057

Bourguignon, M., De Tiège, X., De Beeck, M. O., Van Bogaert, P., Goldman, S., Jousmäki, V., et al. (2013). Primary motor cortex and cerebellum are coupled with the kinematics of observed hand movements. Neuroimage 66, 500-507. doi: 10.1016/j.neuroimage.2012.10.038

Brandes, U. (2001). A faster algorithm for betweenness centrality. J. Math. Soc. 25, 163-177. doi: 10.1080/0022250X.2001.9990249

Brookes, M. J., Woolrich, M., Luckhoo, H., Price, D., Hale, J. R., Stephenson, M. C., et al. (2011). Investigating the electrophysiological basis of resting state networks using magnetoencephalography. Proc. Natl. Acad. Sci. U.S.A. 108, 16783-16788. doi: 10.1073/pnas.1112685108

Brookes, M. J., Woolrich, M. W., and Price, D. (2014). "An Introduction to MEG connectivity measurements," in Magnetoencephalography: From Signals to Dynamical Cortical Networks, eds S. Supek and C. J. Aine (Berlin: Springer), 321-358.
Brown, H. R., and Friston, K. J. (2013). The functional anatomy of attention: a DCM study. Front. Hum. Neurosci. 7:784. doi: 10.3389/fnhum.2013.00784

Bruce, C., Desimone, R., and Gross, C. G. (1981). Visual properties of neurons in a polysensory area in superior temporal sulcus of the macaque. J. Neurophysiol. $46,369-384$

Bruns, A., Eckhorn, R., Jokeit, H., and Ebner, A. (2000). Amplitude envelope correlation detects coupling among incoherent brain signals. Neuroreport 11, 1509-1514. doi: 10.1097/00001756-200005150-00028

Bullmore, E., Fadili, J., Maxim, V., Sendur, L., Whitcher, B., Suckling, J., et al. (2004). Wavelets and functional magnetic resonance imaging of the human brain. Neuroimage 23(Suppl. 1), S234-S249. doi: 10.1016/j.neuroimage.2004.07.012

Bullmore, E., and Sporns, O. (2009). Complex brain networks: graph theoretical analysis of structural and functional systems. Nat. Rev. Neurosci. 10, 186-198. doi: $10.1038 / \mathrm{nrn} 2575$

Bullmore, E. T., Brammer, M. J., Bourlon, P., Alarcon, G., Polkey, C. E., Elwes, R., et al. (1994). Fractal analysis of electroencephalographic signals intracerebrally recorded during 35 epileptic seizures: evaluation of a new method for synoptic visualisation of ictal events. Electroencephalogr. Clin. Neurophysiol. 91, 337-345. doi: 10.1016/0013-4694(94)00181-2

Busk, J., and Galbraith, G. C. (1975). EEG correlates of visual-motor practice in man. Electroencephalogr. Clin. Neurophysiol. 38, 415-422. doi: 10.1016/00134694(75) $90265-5$

Calmels, C., Hars, M., Holmes, P., Jarry, G., and Stam, C. J. (2008). Nonlinear EEG synchronization during observation and execution of simple and complex sequential finger movements. Exp. Brain Res. 190, 389-400. doi: $10.1007 / \mathrm{s} 00221-008-1480-\mathrm{z}$

Calmels, C., Hars, M., Jarry, G., and Stam, C. J. (2010). Non-linear EEG synchronization during observation: effects of instructions and expertise. Psychophysiology 47, 799-808. doi: 10.1111/j.1469-8986.2010.00985.x

Chávez, M., Martinerie, J., and Le Van Quyen, M. (2003). Statistical assessment of nonlinear causality: application to epileptic EEG signals. J. Neurosci. Methods 124, 113-128. doi: 10.1016/S0165-0270(02)00367-9

Cho, J. H., Vorwerk, J., Wolters, C. H., and Knösche, T. R. (2015). Influence of the head model on EEG and MEG source connectivity analyses. Neuroimage 110, 60-77. doi: 10.1016/j.neuroimage.2015.01.043

Cogan, G. B., and Poeppel, D. (2011). A mutual information analysis of neural coding of speech by low-frequency MEG phase information. J. Neurophysiol. 106, 554-563. doi: 10.1152/jn.00075.2011

Cohen, D. (1968). Magnetoencephalography: evidence of magnetic fields produced by alpha-rhythm currents. Science 161, 784-786. doi: 10.1126/science.161.3843.784

Cohen, D. (1972). Magnetoencephalography: detection of the brain's electrical activity with a superconducting magnetometer. Science 175, 664-666. doi: 10.1126/science.175.4022.664

Corbetta, M., Miezin, F. M., Dobmeyer, S., Shulman, G. L., and Petersen, S. E. (1990). Attentional modulation of neural processing of shape, color, and velocity in humans. Science 248, 1556-1559. doi: 10.1126/science.2360050

Craddock, R. C., James, G. A., Holtzheimer, P. E. III, Hu, X. P., and Mayberg, H. S. (2012). A whole brain fMRI atlas generated via spatially constrained spectral clustering. Hum. Brain Mapp. 33, 1914-1928. doi: 10.1002/hbm.21333

Damasio, H., Grabowski, T., Frank, R., Galaburda, A. M., and Damasio, A. R. (1994). The return of Phineas Gage: clues about the brain from the skull of a famous patient. Science 264, 1102-1105. doi: 10.1126/science.8178168

David, O., Cosmelli, D., Hasboun, D., and Garnero, L. (2003). A multitrial analysis for revealing significant corticocortical networks in magnetoencephalography and electroencephalography. Neuroimage 20, 186-201. doi: 10.1016/S10538119(03)00221-0

David, O., Garnero, L., Cosmelli, D., and Varela, F. J. (2002). Estimation of neural dynamics from MEG/EEG cortical current density maps: application to the reconstruction of large-scale cortical synchrony. IEEE Trans. Biomed. Eng. 49, 975-987. doi: 10.1109/TBME.2002.802013

De Pasquale, F., Della Penna, S., Snyder, A. Z., Lewis, C., Mantini, D., Marzetti, L., et al. (2010). Temporal dynamics of spontaneous MEG activity in brain networks. Proc. Natl. Acad. Sci. U.S.A. 107, 6040-6045. doi: 10.1073/pnas.0913863107

Diwakar, M., Huang, M. X., Srinivasan, R., Harrington, D. L., Robb, A., Angeles, A., et al. (2011). Dual-Core Beamformer for obtaining highly 
correlated neuronal networks in MEG. Neuroimage 54, 253-263. doi: 10.1016/j.neuroimage.2010.07.023

Dodel, S., Hermann, J. M., and Geisel, T. (2002). Functional connectivity by crosscorrelation clustering. Neurocomputing 44-46, 1065-1070. doi: 10.1016/S09252312(02)00416-2

Douw, L., Schoonheim, M. M., Landi, D., Van Der Meer, M. L., Geurts, J. J., Reijneveld, J. C., et al. (2011). Cognition is related to resting-state smallworld network topology: an magnetoencephalographic study. Neuroscience 175, 169-177. doi: 10.1016/j.neuroscience.2010.11.039

Downar, J., Crawley, A. P., Mikulis, D. J., and Davis, K. D. (2000). A multimodal cortical network for the detection of changes in the sensory environment. Nat. Neurosci. 3, 277-283. doi: 10.1038/72991

Downing, P. E., Jiang, Y., Shuman, M., and Kanwisher, N. (2001). A cortical area selective for visual processing of the human body. Science 293, 2470-2473. doi: $10.1126 /$ science. 1063414

Evarts, E. V. (1968). Relation of pyramidal tract activity to force exerted during voluntary movement. J. Neurophysiol. 31, 14-27.

Ewald, A., Marzetti, L., Zappasodi, F., Meinecke, F. C., and Nolte, G. (2012). Estimating true brain connectivity from EEG/MEG data invariant to linear and static transformations in sensor space. Neuroimage 60, 476-488. doi: 10.1016/j.neuroimage.2011.11.084

Fein, G., Raz, J., Brown, F. F., and Merrin, E. L. (1988). Common reference coherence data are confounded by power and phase effects. Electroencephalogr. Clin. Neurophysiol. 69, 581-584. doi: 10.1016/0013-4694(88) 90171-X

FitzGerald, T. H., Moran, R. J., Friston, K. J., and Dolan, R. J. (2015). Precision and neuronal dynamics in the human posterior parietal cortex during evidence accumulation. Neuroimage 107, 219-228. doi: 10.1016/j.neuroimage.2014.12.015

Florian, G., Andrew, C., and Pfurtscheller, G. (1998). Do changes in coherence always reflect changes in functional coupling? Electroencephalogr. Clin. Neurophysiol. 106, 87-91.

Freeman, L. C. (1978). Centrality in social network: conceptual clarification. Soc. Netw. 1, 215-239. doi: 10.1016/0378-8733(78)90021-7

Friston, K. J. (1994). Functional and effective connectivity in neuroimaing: a synthesis. Hum. Brain Mapp. 2, 56-78. doi: 10.1002/hbm.460020107

Friston, K. J. (2011). Functional and effective connectivity: a review. Brain Connect. 1, 13-36. doi: 10.1089/brain.2011.0008

Friston, K. J., Frith, C. D., and Frackowiak, R. S. (1993a). Time-dependent changes in effective connectivity measured with PET. Hum. Brain Mapp. 1, 69-79.

Friston, K. J., Frith, C. D., Liddle, P. F., and Frackowiak, R. S. (1993b). Functional connectivity: the principal-component analysis of large (PET) data sets. J. Cereb. Blood Flow Metab. 13, 5-14.

Friston, K. J., Harrison, L., and Penny, W. (2003). Dynamic causal modelling. Neuroimage 19, 1273-1302. doi: 10.1016/S1053-8119(03)00202-7

Friston, K. J., Worsley, K. J., Frackowiak, R. S., Mazziotta, J. C., and Evans, A. C. (1994). Assessing the significance of focal activations using their spatial extent. Hum. Brain Mapp. 1, 210-220. doi: 10.1002/hbm.460010306

Gallagher, H. L., Happé, F., Brunswick, N., Fletcher, P. C., Frith, U., and Frith, C. D. (2000). Reading the mind in cartoons and stories: an fMRI study of 'theory of mind' in verbal and nonverbal tasks. Neuropsychologia 38, 11-21. doi: 10.1016/S0028-3932(99)00053-6

Garrido, M. I., Barnes, G. R., Kumaran, D., Maguire, E. A., and Dolan, R. J. (2015). Ventromedial prefrontal cortex drives hippocampal theta oscillations induced by mismatch computations. Neuroimage 120, 362-370. doi: 10.1016/j.neuroimage.2015.07.016

Gerstein, G. L., Bedenbaugh, P., and Aertsen, M. H. (1989). Neuronal assemblies. IEEE Trans. Biomed. Eng. 36, 4-14. doi: 10.1109/10.16444

Gerstein, G. L., and Perkel, D. H. (1969). Simultaneously recorded trains of action potentials: analysis and functional interpretation. Science 164, 828-830. doi: $10.1126 /$ science. 164.3881 .828

Geweke, J. (1982). Measurement of linear-dependence and feedback between multiple time-series. J. Am. Stat. Assoc. 77, 304-313. doi: 10.1080/01621459.1982.10477803

Gireesh, E. D., and Plenz, D. (2008). Neuronal avalanches organize as nested theta- and beta/gamma-oscillations during development of cortical layer 2/3. Proc. Natl. Acad. Sci. U.S.A. 105, 7576-7581. doi: 10.1073/pnas.0800 537105
Gochin, P. M., Miller, E. K., Gross, C. G., and Gerstein, G. L. (1991). Functional interactions among neurons in inferior temporal cortex of the awake macaque. Exp. Brain Res. 84, 505-516. doi: 10.1007/BF00230962

Gómez, C., Lizier, J. T., Schaum, M., Wollstadt, P., Grutzner, C., Uhlhaas, P., et al. (2014). Reduced predictable information in brain signals in autism spectrum disorder. Front. Neuroinform. 8:9. doi: 10.3389/fninf.2014.00009

Gootjes, L., Bouma, A., Van Strien, J. W., Scheltens, P., and Stam, C. J. (2006). Attention modulates hemispheric differences in functional connectivity: evidence from MEG recordings. Neuroimage 30, 245-253. doi: 10.1016/j.neuroimage.2005.09.015

Gross, J., Kujala, J., Hamalainen, M., Timmermann, L., Schnitzler, A., and Salmelin, R. (2001). Dynamic imaging of coherent sources: studying neural interactions in the human brain. Proc. Natl. Acad. Sci. U.S.A. 98, 694-699. doi: 10.1073/pnas.98.2.694

Gross, J., Kujala, J., Salmelin, R., and Schnitzler, A. (2010). "Noninvasive functional tomographic connectivity analysis with magnetoencephalography," in $M E G$ : An Introduction to Methods, eds P. Hansen, M. L. Kringelbach, and R. Salmelin (New York, NY: Oxford University Press), 216-245.

Gross, J., Pollok, B., Dirks, M., Timmermann, L., Butz, M., and Schnitzler, A. (2005). Task-dependent oscillations during unimanual and bimanual movements in the human primary motor cortex and SMA studied with magnetoencephalography. Neuroimage 26, 91-98. doi: 10.1016/j.neuroimage.2005.01.025

Hagmann, P. (2005). From Diffusion MRI to Brain Connetomics. Ph.D. thesis, École Polytechnique Fédérale de Lausanne (EPFL), Lausanne.

Hagmann, P., Cammoun, L., Gigandet, X., Meuli, R., Honey, C. J., Wedeen, V. J., et al. (2008). Mapping the structural core of human cerebral cortex. PLoS Biol. 6:e159. doi: 10.1371/journal.pbio.0060159

Hagmann, P., Kurant, M., Gigandet, X., Thiran, P., Wedeen, V. J., Meuli, R., et al. (2007). Mapping human whole-brain structural networks with diffusion MRI. PLoS ONE 2:e597. doi: 10.1371/journal.pone.0000597

Hahn, G., Petermann, T., Havenith, M. N., Yu, S., Singer, W., Plenz, D., et al. (2010). Neuronal avalanches in spontaneous activity in vivo. J. Neurophysiol. 104, 3312-3322. doi: 10.1152/jn.00953.2009

Hall, E. L., Woolrich, M. W., Thomaz, C. E., Morris, P. G., and Brookes, M. J. (2013). Using variance information in magnetoencephalography measures of functional connectivity. Neuroimage 67, 203-212. doi: 10.1016/j.neuroimage.2012.11.011

Hämäläinen, M., Hari, R., Ilmoniemi, R. J., Knuutila, J., and Lounasmaa, O.V. (1993). Magnetoencephalography-theory, instrumentation, and applications to noninvasive studies of the working human brain. Rev. Mod. Phys. 65, 413-497. doi: 10.1103/RevModPhys.65.413

Hämäläinen, M. S., and Ilmoniemi, R. J. (1994). Interpreting magnetic fields of the brain: minimum norm estimates. Med. Biol. Eng. Comput. 32, 35-42. doi: 10.1007/BF02512476

Harding, I. H., Yücel, M., Harrison, B. J., Pantelis, C., and Breakspear, M. (2015). Effective connectivity within the frontoparietal control network differentiates cognitive control and working memory. Neuroimage 106, 144-153. doi: 10.1016/j.neuroimage.2014.11.039

Hari, R., and Parkkonen, L. (2015). The brain timewise: how timing shapes and supports brain function. Philos. Trans. R. Soc. Lond. B. Biol. Sci. 370. doi: $10.1098 / \mathrm{rstb} .2014 .0170$

He, F., Billings, S. A., Wei, H. L., and Sarrigiannis, P. G. (2014). A nonlinear causality measure in the frequency domain: nonlinear partial directed coherence with applications to EEG. J. Neurosci. Methods 225, 71-80. doi: 10.1016/j.jneumeth.2014.01.013

Hillebrand, A., and Barnes, G. R. (2002). A quantitative assessment of the sensitivity of whole-head MEG to activity in the adult human cortex. Neuroimage 16, 638-650. doi: 10.1006/nimg.2002.1102

Hinrichs, H., Heinze, H. J., and Schoenfeld, M. A. (2006). Causal visual interactions as revealed by an information theoretic measure and fMRI. Neuroimage 31, 1051-1060. doi: 10.1016/j.neuroimage.2006.01.038

Hinrichs, H., Noesselt, T., and Heinze, H. J. (2008). Directed information flow: a model free measure to analyze causal interactions in event related EEG-MEGexperiments. Hum. Brain Mapp. 29, 193-206. doi: 10.1002/hbm.20382

Hipp, J. F., Hawellek, D. J., Corbetta, M., Siegel, M., and Engel, A. K. (2012). Large-scale cortical correlation structure of spontaneous oscillatory activity. Nat. Neurosci. 15, 884-890. doi: 10.1038/nn.3101 
Hipp, J. F., and Siegel, M. (2015). BOLD fMRI correlation reflects frequency-specific neuronal correlation. Curr. Biol. 25, 1368-1374. doi: 10.1016/j.cub.2015.03.049

Hoechstetter, K., Bornfleth, H., Weckesser, D., Ille, N., Berg, P., and Scherg, M. (2004). BESA source coherence: a new method to study cortical oscillatory coupling. Brain Topogr. 16, 233-238. doi: 10.1023/B:BRAT.0000032857.55223.5d

Honey, C. J., and Sporns, O. (2008). Dynamical consequences of lesions in cortical networks. Hum. Brain Mapp. 29, 802-809. doi: 10.1002/hbm.20579

Hsiao, F. J., Yu, H. Y., Chen, W. T., Kwan, S. Y., Chen, C., Yen, D. J., et al. (2015). Increased intrinsic connectivity of the default mode network in temporal lobe epilepsy: evidence from resting-state MEG recordings. PLoS ONE 10:e0128787. doi: 10.1371 /journal.pone. 0128787

Huang, M. X., Huang, C. W., Robb, A., Angeles, A., Nichols, S. L., Baker, D. G., et al. (2014). MEG source imaging method using fast L1 minimum-norm and its applications to signals with brain noise and human resting-state source amplitude images. Neuroimage 84, 585-604. doi: 10.1016/j.neuroimage.2013.09.022

Hubel, D. H., and Wiesel, T. N. (1968). Receptive fields and functional architecture of monkey striate cortex. J. Physiol. 195, 215-243. doi: 10.1113/jphysiol.1968.sp008455

Humphries, M. D., and Gurney, K. (2008). Network 'small-world-ness': a quantitative method for determining canonical network equivalence. PLoS ONE 3:e0002051. doi: 10.1371/journal.pone.0002051

Iacoboni, M., Molnar-Szakacs, I., Gallese, V., Buccino, G., Mazziotta, J. C., and Rizzolatti, G. (2005). Grasping the intentions of others with one's own mirror neuron system. PLoS Biol. 3:e79. doi: 10.1371/journal.pbio.0030079

Ilmoniemi, R. J., Virtanen, J., Ruohonen, J., Karhu, J., Aronen, H. J., Naatanen, R., et al. (1997). Neuronal responses to magnetic stimulation reveal cortical reactivity and connectivity. Neuroreport 8, 3537-3540. doi: 10.1097/00001756199711100-00024

Ioannides, A. A., Bolton, J. P. R., and Clarke, C. J. S. (1990). Continuous probabilistic solutions to the biomagnetic inverse problem. Inverse Probl. 6, 523-542. doi: 10.1088/0266-5611/6/4/005

Ioannides, A. A., Liu, L. C., Kwapien, J., Drozdz, S., and Streit, M. (2000). Coupling of regional activations in a human brain during an object and face affect recognition task. Hum. Brain Mapp. 11, 77-92. doi: 10.1002/10970193(200010)11:2<77::AID-HBM20>3.0.CO;2-0

Ioannides, A. A., Liu, L., Poghosyan, V., Saridis, G. A., Gjedde, A., Ptito, M., et al. (2013). MEG reveals a fast pathway from somatosensory cortex to occipital areas via posterior parietal cortex in a blind subject. Front. Hum. Neurosci. 7:429. doi: 10.3389/fnhum.2013.00429

Ioannides, A. A., Poghosyan, V., Dammers, J., and Streit, M. (2004). Real-time neural activity and connectivity in healthy individuals and schizophrenia patients. Neuroimage 23, 473-482. doi: 10.1016/j.neuroimage.2004.06.023

Iturria-Medina, Y., Canales-Rodríguez, E. J., Melie-García, L., Valdés-Hernández, P. A., Martínez-Montes, E., Alemán-Gómez, Y., et al. (2007). Characterizing brain anatomical connections using diffusion weighted MRI and graph theory. Neuroimage 36, 645-660. doi: 10.1016/j.neuroimage.2007.02.012

Jin, S. H., Jeong, W., Lee, D. S., Jeon, B. S., and Chung, C. K. (2014). Preserved highcentrality hubs but efficient network reorganization during eyes-open state compared with eyes-closed resting state: an MEG study. J. Neurophysiol. 111, 1455-1465. doi: 10.1152/jn.00585.2013

Jin, S. H., Jeong, W., Seol, J., Kwon, J., and Chung, C. K. (2013). Functional cortical hubs in the eyes-closed resting human brain from an electrophysiological perspective using magnetoencephalography. PLoS ONE 8:e68192. doi: 10.1371/journal.pone.0068192

Jin, S. H., Seol, J., Kim, J. S., and Chung, C. K. (2011). How reliable are the functional connectivity networks of MEG in resting states? J. Neurophysiol. 106, 2888-2895. doi: 10.1152/jn.00335.2011

Kakigi, R., Hoshiyama, M., Shimojo, M., Naka, D., Yamasaki, H., Watanabe, S., et al. (2000). The somatosensory evoked magnetic fields. Prog. Neurobiol. 61, 495-523. doi: 10.1016/S0301-0082(99)00063-5

Kaminski, M. J., and Blinowska, K. J. (1991). A new method of the description of the information flow in the brain structures. Biol. Cybern. 65, 203-210. doi: 10.1007/BF00198091
Kamitake, T., Harashima, H., and Miyakawa, H. (1984). A time-series analysis method based on the directed transformation. Electon. Commun. Jpn. 67, 1-9. doi: $10.1002 /$ ecja.4400670602

Kanwisher, N., McDermott, J., and Chun, M. M. (1997). The fusiform face area: a module in human extrastriate cortex specialized for face perception. J. Neurosci. $17,4302-4311$

Kida, T., and Kakigi, R. (2013). Task-related changes in functional properties of the human brain network underlying attentional control. PLoS ONE 8:e79023. doi: 10.1371/journal.pone.0079023

Kida, T., Tanaka, E., and Kakigi, R. (2013). "Task-related changes in functional properties of the human brain network," in Neuroscience Meeting 2013. Program No. 763.18 (San Diego, CA).

Kintali, S. (2008). Betweenness centrality: algorithms and lower bounds. arXiv 0809.1906v11.

Kitzbichler, M. G., Smith, M. L., Christensen, S. R., and Bullmore, E. (2009). Broadband criticality of human brain network synchronization. PLoS Comput. Biol. 5:e1000314. doi: 10.1371/journal.pcbi.1000314

Klingner, C. M., Brodoehl, S., Huonker, R., Götz, T., Baumann, L., and Witte, O. W. (2015). Parallel processing of somatosensory information: evidence from dynamic causal modeling of MEG data. Neuroimage 118, 193-198. doi: 10.1016/j.neuroimage.2015.06.028

Kraskov, A., Stögbauer, H., and Grassberger, P. (2004). Estimating mutual information. Phys. Rev. E Stat. Nonlin. Soft Matter Phys. 69:066138. doi: 10.1103/PhysRevE.69.066138

Lachaux, J. P., Rodriguez, E., Martinerie, J., and Varela, F. J. (1999). Measuring phase synchrony in brain signals. Hum. Brain Mapp. 8, 194-208.

Langers, D. R., Backes, W. H., and Van Dijk, P. (2007). Representation of lateralization and tonotopy in primary versus secondary human auditory cortex. Neuroimage 34, 264-273. doi: 10.1016/j.neuroimage.2006.09.002

Latora, V., and Marchiori, M. (2001). Efficient behavior of small-world networks. Phys. Rev. Lett. 87:198701. doi: 10.1103/PhysRevLett.87.198701

Leicht, E. A., and Newman, M. E. (2008). Community structure in directed networks. Phys. Rev. Lett. 100:118703. doi: 10.1103/PhysRevLett.100.118703

Leopold, D. A., Murayama, Y., and Logothetis, N. K. (2003). Very slow activity fluctuations in monkey visual cortex: implications for functional brain imaging. Cereb. Cortex 13, 422-433. doi: 10.1093/cercor/13.4.422

Lin, F. H., Witzel, T., Hämäläinen, M. S., Dale, A. M., Belliveau, J. W., and Stufflebeam, S. M. (2004). Spectral spatiotemporal imaging of cortical oscillations and interactions in the human brain. Neuroimage 23, 582-595. doi: 10.1016/j.neuroimage.2004.04.027

Linkenkaer-Hansen, K., Nikouline, V. V., Palva, J. M., and Ilmoniemi, R. J. (2001). Long-range temporal correlations and scaling behavior in human brain oscillations. J. Neurosci. 21, 1370-1377.

Lizier, J. T., Heinzle, J., Horstmann, A., Haynes, J. D., and Prokopenko, M. (2011). Multivariate information-theoretic measures reveal directed information structure and task relevant changes in fMRI connectivity. J. Comput. Neurosci. 30, 85-107. doi: 10.1007/s10827-010-0271-2

Lobier, M., Siebenhühner, F., Palva, S., and Palva, J. M. (2014). Phase transfer entropy: a novel phase-based measure for directed connectivity in networks coupled by oscillatory interactions. Neuroimage 85(Pt 2), 853-872. doi: 10.1016/j.neuroimage.2013.08.056

Lombardi, F., Herrmann, H. J., Perrone-Capano, C., Plenz, D., and De Arcangelis, L. (2012). Balance between excitation and inhibition controls the temporal organization of neuronal avalanches. Phys. Rev. Lett. 108:228703. doi: 10.1103/PhysRevLett.108.228703

Luce, R. D., and Perry, A. D. (1949). A method of matrix analysis of group structure. Psychometrika 14, 95-116. doi: 10.1007/BF02289146

Luckhoo, H., Hale, J. R., Stokes, M. G., Nobre, A. C., Morris, P. G., Brookes, M. J., et al. (2012). Inferring task-related networks using independent component analysis in magnetoencephalography. Neuroimage 62, 530-541. doi: 10.1016/j.neuroimage.2012.04.046

Makeig, S., Debener, S., Onton, J., and Delorme, A. (2004a). Mining event-related brain dynamics. Trends Cogn. Sci. 8, 204-210. doi: 10.1016/j.tics.2004.03.008

Makeig, S., Delorme, A., Westerfield, M., Jung, T. P., Townsend, J., Courchesne, E., et al. (2004b). Electroencephalographic brain dynamics following manually responded visual targets. PLoS Biol. 2:e176. doi: 10.1371/journal. pbio.0020176 
Martuzzi, R., Van Der Zwaag, W., Farthouat, J., Gruetter, R., and Blanke, O. (2014). Human finger somatotopy in areas $3 \mathrm{~b}, 1$, and 2: a $7 \mathrm{~T}$ fMRI study using a natural stimulus. Hum. Brain Mapp. 35, 213-226. doi: 10.1002/hbm.22172

Marty, B., Bourguignon, M., Jousmäki, V., Wens, V., Op De Beeck, M., Van Bogaert, P., et al. (2015). Cortical kinematic processing of executed and observed goal-directed hand actions. Neuroimage 119, 221-228. doi: 10.1016/j.neuroimage.2015.06.064

Matsumoto, R., Nair, D. R., Ikeda, A., Fumuro, T., Lapresto, E., Mikuni, N., et al. (2012). Parieto-frontal network in humans studied by cortico-cortical evoked potential. Hum. Brain Mapp. 33, 2856-2872. doi: 10.1002/hbm.21407

Maxim, V., Sendur, L., Fadili, J., Suckling, J., Gould, R., Howard, R., et al. (2005). Fractional Gaussian noise, functional MRI and Alzheimer's disease. Neuroimage 25, 141-158. doi: 10.1016/j.neuroimage.2004.10.044

Michel, C. M., and Murray, M. M. (2012). Towards the utilization of EEG as a brain imaging tool. Neuroimage 61, 371-385. doi: 10.1016/j.neuroimage.2011.12.039

Milo, R., Shen-Orr, S., Itzkovitz, S., Kashtan, N., Chklovskii, D., and Alon, U. (2002). Network motifs: simple building blocks of complex networks. Science 298, 824-827. doi: 10.1126/science.298.5594.824

Mima, T., Matsuoka, T., and Hallett, M. (2000). Functional coupling of human right and left cortical motor areas demonstrated with partial coherence analysis. Neurosci. Lett. 287, 93-96. doi: 10.1016/S0304-3940(00)01165-4

Mohseni, H. R., Kringelbach, M. L., Woolrich, M. W., Baker, A., Aziz, T. Z., and Probert-Smith, P. (2014). Non-Gaussian probabilistic MEG source localisation based on kernel density estimation. Neuroimage 87, 444-464. doi: 10.1016/j.neuroimage.2013.09.012

Morishima, Y., Akaishi, R., Yamada, Y., Okuda, J., Toma, K., and Sakai, K. (2009). Task-specific signal transmission from prefrontal cortex in visual selective attention. Nat. Neurosci. 12, 85-91. doi: 10.1038/nn.2237

Mormann, F., Lehnertz, K., David, P., and Elger, C. E. (2000). Mean phase coherence as a measure for phase synchronization and its application to the EEG of epilepsy patients. Physica D 144, 358-369. doi: 10.1016/S01672789(00)00087-7

Newman, M. E. (2006). Modularity and community structure in networks. Proc. Natl. Acad. Sci. U.S.A. 103, 8577-8582. doi: 10.1073/pnas.0601602103

Nir, Y., Mukamel, R., Dinstein, I., Privman, E., Harel, M., Fisch, L., et al. (2008). Interhemispheric correlations of slow spontaneous neuronal fluctuations revealed in human sensory cortex. Nat. Neurosci. 11, 1100-1108. doi: $10.1038 / \mathrm{nn} .2177$

Noguchi, Y., Watanabe, E., and Sakai, K. L. (2003). An event-related optical topography study of cortical activation induced by singlepulse transcranial magnetic stimulation. Neuroimage 19, 156-162. doi: 10.1016/S1053-8119(03)00054-5

Nolte, G., Bai, O., Wheaton, L., Mari, Z., Vorbach, S., and Hallett, M. (2004). Identifying true brain interaction from EEG data using the imaginary part of coherency. Clin. Neurophysiol. 115, 2292-2307. doi: 10.1016/j.clinph.2004.04.029

Nolte, G., and Müller, K. R. (2010). Localizing and estimating causal relations of interacting brain rhythms. Front. Hum. Neurosci. 4:209. doi: 10.3389/fnhum.2010.00209

Nolte, G., Ziehe, A., Kramer, N., Popescu, F., and Muller, K. R. (2008a). "Comparison of granger causality and phase slope index," in JMLR Workshop and Conference, eds I. Guyon, D. Janzing, and B. Scholkopf (British Columbia), 267-276.

Nolte, G., Ziehe, A., Nikulin, V. V., Schlögl, A., Krämer, N., Brismar, T., et al. (2008b). Robustly estimating the flow direction of information in complex physical systems. Phys. Rev. Lett. 100, 234101. doi: 10.1103/PhysRevLett.100.234101

Nunez, P. L., and Srinivasan, R. (2006). Electric Fields of the Brain: The Neurophysics of EEG. New York, NY: Oxford Universitiy Press.

Ogawa, S., Lee, T. M., Kay, A. R., and Tank, D. W. (1990). Brain magnetic resonance imaging with contrast dependent on blood oxygenation. Proc. Natl. Acad. Sci. U.S.A. 87, 9868-9872. doi: 10.1073/pnas.87.24.9868

Owen, J. P., Wipf, D. P., Attias, H. T., Sekihara, K., and Nagarajan, S. S. (2009). Accurate reconstruction of brain activity and functional connectivity from noisy MEG data. Conf. Proc. IEEE Eng. Med. Biol. Soc. 2009, 65-68. doi: 10.1109/iembs.2009.5335005

Owen, J. P., Wipf, D. P., Attias, H. T., Sekihara, K., and Nagarajan, S. S. (2012). Performance evaluation of the Champagne source reconstruction algorithm on simulated and real M/EEG data. Neuroimage 60, 305-323. doi: 10.1016/j.neuroimage.2011.12.027

Palla, G., Derényi, I., Farkas, I., and Vicsek, T. (2005). Uncovering the overlapping community structure of complex networks in nature and society. Nature 435, 814-818. doi: $10.1038 /$ nature 03607

Palva, J. M., Zhigalov, A., Hirvonen, J., Korhonen, O., Linkenkaer-Hansen, K., and Palva, S. (2013). Neuronal long-range temporal correlations and avalanche dynamics are correlated with behavioral scaling laws. Proc. Natl. Acad. Sci. U.S.A. 110, 3585-3590. doi: 10.1073/pnas.1216855110

Pantev, C., Hoke, M., Lütkenhöner, B., and Lehnertz, K. (1989). Tonotopic organization of the auditory cortex: pitch versus frequency representation. Science 246, 486-488. doi: 10.1126/science.2814476

Paraskevopoulos, E., Kraneburg, A., Herholz, S. C., Bamidis, P. D., and Pantev, C. (2015). Musical expertise is related to altered functional connectivity during audiovisual integration. Proc. Natl. Acad. Sci. U.S.A. 112, 12522-12527. doi: $10.1073 /$ pnas. 1510662112

Park, H., Ince, R. A., Schyns, P. G., Thut, G., and Gross, J. (2015). Frontal top-down signals increase coupling of auditory low-frequency oscillations to continuous speech in human listeners. Curr. Biol. 25, 1649-1653. doi: 10.1016/j.cub.2015.04.049

Parlitz, U., Junge, L., Lauterborn, W., and Kocarev, L. (1996). Experimental observation of phase synchronization. Phys. Rev. E Stat. Phys. Plasmas Fluids Relat. Interdiscip. Topics 54, 2115-2117. doi: 10.1103/physreve.54.2115

Parvizi, J., Jacques, C., Foster, B. L., Witthoft, N., Rangarajan, V., Weiner, K. S., et al. (2012). Electrical stimulation of human fusiform face-selective regions distorts face perception. J. Neurosci. 32, 14915-14920. doi: 10.1523/JNEUROSCI.260912.2012

Paus, T., Jech, R., Thompson, C. J., Comeau, R., Peters, T., and Evans, A. C. (1997). Transcranial magnetic stimulation during positron emission tomography: a new method for studying connectivity of the human cerebral cortex. J. Neurosci. $17,3178-3184$.

Penfield, W., and Rasmussen, T. (1950). The Cerebral Cortex of Man: A Clinical Study of Localization of Function. New York, NY: Macmillan.

Pereda, E., Rial, R., Gamundi, A., and Gonzalez, J. (2001). Assessment of changing interdependencies between human electroencephalograms using nonlinear methods. Physica D 148, 147-158. doi: 10.1016/S0167-2789(00)00190-1

Petermann, T., Thiagarajan, T. C., Lebedev, M. A., Nicolelis, M. A., Chialvo, D. R., and Plenz, D. (2009). Spontaneous cortical activity in awake monkeys composed of neuronal avalanches. Proc. Natl. Acad. Sci. U.S.A. 106, 15921-15926. doi: 10.1073/pnas.0904089106

Poghosyan, V., and Ioannides, A. A. (2008). Attention modulates earliest responses in the primary auditory and visual cortices. Neuron 58, 802-813. doi: 10.1016/j.neuron.2008.04.013

Poil, S. S., Van Ooyen, A., and Linkenkaer-Hansen, K. (2008). Avalanche dynamics of human brain oscillations: relation to critical branching processes and temporal correlations. Hum. Brain Mapp. 29, 770-777. doi: 10.1002/hbm. 20590

Pool, E. M., Rehme, A. K., Fink, G. R., Eickhoff, S. B., and Grefkes, C. (2014). Handedness and effective connectivity of the motor system. Neuroimage 99, 451-460. doi: 10.1016/j.neuroimage.2014.05.048

Power, J. D., Cohen, A. L., Nelson, S. M., Wig, G. S., Barnes, K. A., Church, J. A., et al. (2011). Functional network organization of the human brain. Neuron 72, 665-678. doi: 10.1016/j.neuron.2011.09.006

Puce, A., Allison, T., Asgari, M., Gore, J. C., and McCarthy, G. (1996). Differential sensitivity of human visual cortex to faces, letterstrings, and textures: a functional magnetic resonance imaging study. J. Neurosci. 16, 5205-5215. doi: 10.1016/s1053-8119(96)80364-8

Quiroga, R. Q., Reddy, L., Kreiman, G., Koch, C., and Fried, I. (2005). Invariant visual representation by single neurons in the human brain. Nature 435 , 1102-1107. doi: 10.1038 /nature 03687

Rappelsberger, P. (1989). The reference problem and mapping of coherence: a simulation study. Brain Topogr. 2, 63-72. doi: 10.1007/BF01128844

Regenbogen, C., Habel, U., and Kellermann, T. (2013). Connecting multimodality in human communication. Front. Hum. Neurosci. 7:754. doi: 10.3389/fnhum.2013.00754

Ritterband-Rosenbaum, A., Nielsen, J. B., and Christensen, M. S. (2014). Sense of agency is related to gamma band coupling in an inferior parietal-preSMA circuitry. Front. Hum. Neurosci. 8:510. doi: 10.3389/fnhum.2014.00510 
Robinson, S. E., and Varba, J. (1999). "Functional neuroimaging by synthetic aperture Magnetometry (SAM)," in Recent Advances in Biomagnetism, eds T. Yoshimoto (Sendai: Tohoku University Press), 302-305.

Roland, P. E., Larsen, B., Lassen, N. A., and Skinhøj, E. (1980). Supplementary motor area and other cortical areas in organization of voluntary movements in man. J. Neurophysiol. 43, 118-136.

Romani, G. L., Williamson, S. J., and Kaufman, L. (1982). Tonotopic organization of the human auditory cortex. Science 216, 1339-1340. doi: $10.1126 /$ science. 7079770

Rosenblum, M. G., Pikovsky, A. S., and Kurths, J. (1996). Phase synchronization of chaotic oscillators. Phys. Rev. Lett. 76, 1804-1807. doi: 10.1103/PhysRevLett.76.1804

Roulston, M. S. (1999). Estimating the errors on measured entropy and mutual information. Physica D 125, 285-294. doi: 10.1016/S0167-2789(98) 00269-3

Rubinov, M., and Sporns, O. (2010). Complex network measures of brain connectivity: uses and interpretations. Neuroimage 52, 1059-1069. doi: 10.1016/j.neuroimage.2009.10.003

Rudrauf, D., Douiri, A., Kovach, C., Lachaux, J. P., Cosmelli, D., Chavez, M., et al. (2006). Frequency flows and the time-frequency dynamics of multivariate phase synchronization in brain signals. Neuroimage 31, 209-227. doi: 10.1016/j.neuroimage.2005.11.021

Rulkov, N. F., Sushchik, M. M., Tsimring, L. S., and Abarbanel, H. D. I. (1995). Generalized synchronization of chaos in directionally coupled chaotic systems. Phys. Rev. E 51, 980-994. doi: 10.1103/PhysRevE.51.980

Sabidussi, G. (1966). The centraity index of a graph. Psychometrika 31, 582-603. doi: $10.1007 / \mathrm{BF} 02289527$

Saito, Y., and Harashima, H. (1981). “Tracking of information within multichannel EEG record causal analysis in EEG," in Recent Advances in EEG and EMG Data Processing, eds N. Yamaguchi and K. Fujisawa (New York, NY: Elsevier/NorthHolland Biomedical Press), 133-146.

Sander, T. H., Bock, A., Leistner, S., Kuhn, A., and Trahms, L. (2010). Coherence and imaginary part of coherency identifies cortico-muscular and corticothalamic coupling. Conf. Proc. IEEE Eng. Med. Biol. Soc. 2010, 1714-1717. doi: 10.1109/iembs.2010.5626851

Sarvas, J. (1987). Basic mathematical and electromagnetic concepts of the biomagnetic inverse problem. Phys. Med. Biol. 32, 11-22. doi: 10.1088/00319155/32/1/004

Scheeringa, R., Fries, P., Petersson, K. M., Oostenveld, R., Grothe, I., Norris, D. G., et al. (2011). Neuronal dynamics underlying high- and low-frequency EEG oscillations contribute independently to the human BOLD signal. Neuron 69, 572-583. doi: 10.1016/j.neuron.2010.11.044

Schepers, I. M., Hipp, J. F., Schneider, T. R., Röder, B., and Engel, A. K. (2012). Functionally specific oscillatory activity correlates between visual and auditory cortex in the blind. Brain 135, 922-934. doi: 10.1093/brain/ aws 014

Schiff, S. J., So, P., Chang, T., Burke, R. E., and Sauer, T. (1996). Detecting dynamical interdependence and generalized synchrony through mutual prediction in a neural ensemble. Phys. Rev. E 54, 6708-6724. doi: 10.1103/PhysRevE.54.6708

Schoffelen, J. M., Oostenveld, R., and Fries, P. (2008). Imaging the human motor system's beta-band synchronization during isometric contraction. Neuroimage 41, 437-447. doi: 10.1016/j.neuroimage.2008.01.045

Schreiber, T. (2000). Measuring information transfer. Phys. Rev. Lett. 85, 461-464. doi: 10.1103/PhysRevLett.85.461

Sekihara, K., and Nagarajan, S. S. (2013). Residual coherence and residual envelope correlation in MEG/EEG source-space connectivity analysis. Conf. Proc. IEEE Eng. Med. Biol. Soc. 2013, 4414-4417. doi: 10.1109/embc.2013. 6610525

Sekihara, K., Nagarajan, S. S., Poeppel, D., Marantz, A., and Miyashita, Y. (2001). Reconstructing spatio-temporal activities of neural sources using an MEG vector beamformer technique. IEEE Trans. Biomed. Eng. 48, 760-771. doi: $10.1109 / 10.930901$

Sekihara, K., Owen, J. P., Trisno, S., and Nagarajan, S. S. (2011). Removal of spurious coherence in MEG source-space coherence analysis. IEEE Trans. Biomed. Eng. 58, 3121-3129. doi: 10.1109/TBME.2011.2162514

Sereno, M. I., Dale, A. M., Reppas, J. B., Kwong, K. K., Belliveau, J. W., Brady, T. J., et al. (1995). Borders of multiple visual areas in humans revealed by functional magnetic resonance imaging. Science 268, 889-893. doi: $10.1126 /$ science. 7754376

Shew, W. L., and Plenz, D. (2013). The functional benefits of criticality in the cortex. Neuroscientist 19, 88-100. doi: 10.1177/1073858412445487

Shriki, O., Alstott, J., Carver, F., Holroyd, T., Henson, R. N., Smith, M. L., et al. (2013). Neuronal avalanches in the resting MEG of the human brain. J. Neurosci. 33, 7079-7090. doi: 10.1523/JNEUROSCI.4286-12.2013

Siebner, H. R., Bergmann, T. O., Bestmann, S., Massimini, M., JohansenBerg, H., Mochizuki, H., et al. (2009). Consensus paper: combining transcranial stimulation with neuroimaging. Brain Stimul. 2, 58-80. doi: 10.1016/j.brs.2008.11.002

Siegel, M., Donner, T. H., Oostenveld, R., Fries, P., and Engel, A. K. (2008). Neuronal synchronization along the dorsal visual pathway reflects the focus of spatial attention. Neuron 60, 709-719. doi: 10.1016/j.neuron.2008.09.010

Sklar, B., Hanley, J., and Simmons, W. W. (1972). An EEG experiment aimed toward identifying dyslexic children. Nature 240, 414-416. doi: $10.1038 / 240414 \mathrm{a} 0$

Smit, D. J., De Geus, E. J., Van De Nieuwenhuijzen, M. E., Van Beijsterveldt, C. E., Van Baal, G. C., Mansvelder, H. D., et al. (2011). Scale-free modulation of resting-state neuronal oscillations reflects prolonged brain maturation in humans. J. Neurosci. 31, 13128-13136. doi: 10.1523/JNEUROSCI.1678-11.2011

Smit, D. J., Linkenkaer-Hansen, K., and De Geus, E. J. (2013). Long-range temporal correlations in resting-state alpha oscillations predict human timingerror dynamics. J. Neurosci. 33, 11212-11220. doi: 10.1523/JNEUROSCI.281612.2013

Sporns, O. (2013). The human connectome: origins and challenges. Neuroimage 80, 53-61. doi: 10.1016/j.neuroimage.2013.03.023

Sporns, O., and Bullmore, E. T. (2014). From connections to function: the mouse brain connectome atlas. Cell 157, 773-775. doi: 10.1016/j.cell.2014.04.023

Sporns, O., Tononi, G., and Kötter, R. (2005). The human connectome: a structural description of the human brain. PLoS Comput. Biol. 1:e42. doi: 10.1371/journal.pcbi.0010042

Stam, C. J. (2004). Functional connectivity patterns of human magnetoencephalographic recordings: a 'small-world' network? Neurosci. Lett. 355, 25-28. doi: 10.1016/j.neulet.2003.10.063

Stam, C. J. (2014). Modern network science of neurological disorders. Nat. Rev. Neurosci. 15, 683-695. doi: 10.1038/nrn3801

Stam, C. J., De Haan, W., Daffertshofer, A., Jones, B. F., Manshanden, I., Van Cappellen Van Walsum, A. M., et al. (2009). Graph theoretical analysis of magnetoencephalographic functional connectivity in Alzheimer's disease. Brain 132, 213-224. doi: 10.1093/brain/awn262

Stam, C. J., Jones, B. F., Manshanden, I., Van Walsum, A. M. V., Montez, T., Verbunt, J. P. A., et al. (2006). Magnetoencephalographic evaluation of restingstate functional connectivity in Alzheimer's disease. Neuroimage 32, 1335-1344. doi: 10.1016/j.neuroimage.2006.05.033

Stam, C. J., Jones, B. F., Nolte, G., Breakspear, M., and Scheltens, P. (2007a). Smallworld networks and functional connectivity in Alzheimer's disease. Cereb. Cortex 17, 92-99. doi: 10.1093/cercor/bhj127

Stam, C. J., Nolte, G., and Daffertshofer, A. (2007b). Phase lag index: assessment of functional connectivity from multi channel EEG and MEG with diminished bias from common sources. Hum. Brain Mapp. 28, 1178-1193. doi: $10.1002 / \mathrm{hbm} .20346$

Stam, C. J., and Van Dijk, B. W. (2002). Synchronization likelihood: an unbiased measure of generalized synchronization in multivariate data sets. Physica D 163, 236-251. doi: 10.1016/S0167-2789(01)00386-4

Stephan, K. E., Kasper, L., Harrison, L. M., Daunizeau, J., Den Ouden, H. E., Breakspear, M., et al. (2008). Nonlinear dynamic causal models for fMRI. Neuroimage 42, 649-662. doi: 10.1016/j.neuroimage.2008.04.262

Suckling, J., Wink, A. M., Bernard, F. A., Barnes, A., and Bullmore, E. (2008). Endogenous multifractal brain dynamics are modulated by age, cholinergic blockade and cognitive performance. J. Neurosci. Methods 174, 292-300. doi: 10.1016/j.jneumeth.2008.06.037

Supek, S., and Aine, C. J. (2014). Magnetoencephalography: From Signals to Dynamic Cortical Networks. Berlin: Springer.

Supek, S., Aine, C. J., Ranken, D., Best, E., Flynn, E. R., and Wood, C. C. (1999). Single vs. paired visual stimulation: superposition of early neuromagnetic responses and retinotopy in extrastriate cortex in humans. Brain Res. 830, 43-55. doi: 10.1016/S0006-8993(99)01316-5 
Takei, T., and Seki, K. (2008). Spinomuscular coherence in monkeys performing a precision grip task. J. Neurophysiol. 99, 2012-2020. doi: 10.1152/jn.01181.2007

Tass, P. A., Fieseler, T., Dammers, J., Dolan, K., Morosan, P., Majtanik, M., et al. (2003). Synchronization tomography: a method for three-dimensional localization of phase synchronized neuronal populations in the human brain using magnetoencephalography. Phys. Rev. Lett. 90:088101. doi: 10.1103/PhysRevLett.90.088101

Thioux, M., and Keysers, C. (2015). Object visibility alters the relative contribution of ventral visual stream and mirror neuron system to goal anticipation during action observation. Neuroimage 105, 380-394. doi: 10.1016/j.neuroimage.2014.10.035

Tsao, D. Y., Freiwald, W. A., Tootell, R. B., and Livingstone, M. S. (2006). A cortical region consisting entirely of face-selective cells. Science 311, 670-674. doi: $10.1126 /$ science.1119983

Tsao, D. Y., Moeller, S., and Freiwald, W. A. (2008). Comparing face patch systems in macaques and humans. Proc. Natl. Acad. Sci. U.S.A. 105, 19514-19519. doi: 10.1073/pnas.0809662105

Uutela, K., Hämäläinen, M., and Somersalo, E. (1999). Visualization of magnetoencephalographic data using minimum current estimates. Neuroimage 10, 173-180. doi: 10.1006/nimg.1999.0454

Vaina, L. M. (1989). Selective impairment of visual motion interpretation following lesions of the right occipito-parietal area in humans. Biol. Cybern. 61, 347-359. doi: 10.1007/BF00200800

Van Wingerden, M., Vinck, M., Lankelma, J., and Pennartz, C. M. (2010). Theta-band phase locking of orbitofrontal neurons during reward expectancy. J. Neurosci. 30, 7078-7087. doi: 10.1523/JNEUROSCI.3860-09.2010

Vicente, R., Wibral, M., Lindner, M., and Pipa, G. (2011). Transfer entropy-a model-free measure of effective connectivity for the neurosciences. J. Comput. Neurosci. 30, 45-67. doi: 10.1007/s10827-010-0262-3

Vinck, M., Oostenveld, R., Van Wingerden, M., Battaglia, F., and Pennartz, C. M. (2011). An improved index of phase-synchronization for electrophysiological data in the presence of volume-conduction, noise and sample-size bias. Neuroimage 55, 1548-1565. doi: 10.1016/j.neuroimage.2011.01.055

Volz, L. J., Eickhoff, S. B., Pool, E. M., Fink, G. R., and Grefkes, C. (2015). Differential modulation of motor network connectivity during movements of the upper and lower limbs. Neuroimage 119, 44-53. doi: 10.1016/j.neuroimage.2015.05.101

Walter, D. O. (1963). Spectral analysis for electroencephalograms: mathematical determination of neurophysiological relationships from records of limited duration. Exp. Neurol. 8, 155-181. doi: 10.1016/0014-4886(63)90042-6

Wandell, B. A., Dumoulin, S. O., and Brewer, A. A. (2007). Visual field maps in human cortex. Neuron 56, 366-383. doi: 10.1016/j.neuron.2007.10.012
Wang, J. X., Bartolotti, J., Amaral, L. A., and Booth, J. R. (2013). Changes in task-related functional connectivity across multiple spatial scales are related to reading performance. PLoS ONE 8:e59204. doi: 10.1371/journal.pone.0059204

Wasserman, S., and Faust, K. (1994). Social Network Analysis: Methods and Applications. Cambridge: Cambridge University Press.

Watts, D. J., and Strogatz, S. H. (1998). Collective dynamics of 'small-world' networks. Nature 393, 440-442. doi: 10.1038/30918

Wibral, M., Rahm, B., Rieder, M., Lindner, M., Vicente, R., and Kaiser, J. (2011). Transfer entropy in magnetoencephalographic data: quantifying information flow in cortical and cerebellar networks. Prog. Biophys. Mol. Biol. 105, 80-97. doi: 10.1016/j.pbiomolbio.2010.11.006

Wink, A. M., Bullmore, E., Barnes, A., Bernard, F., and Suckling, J. (2008). Monofractal and multifractal dynamics of low frequency endogenous brain oscillations in functional MRI. Hum. Brain Mapp. 29, 791-801. doi: 10.1002/hbm.20593

Wipf, D. P., Owen, J. P., Attias, H. T., Sekihara, K., and Nagarajan, S. S. (2010) Robust Bayesian estimation of the location, orientation, and time course of multiple correlated neural sources using MEG. Neuroimage 49, 641-655. doi: 10.1016/j.neuroimage.2009.06.083

Yang, H., Shew, W. L., Roy, R., and Plenz, D. (2012). Maximal variability of phase synchrony in cortical networks with neuronal avalanches. J. Neurosci. 32, 1061-1072. doi: 10.1523/JNEUROSCI.2771-11.2012

Youssofzadeh, V., Prasad, G., and Wong-Lin, K. (2015). On self-feedback connectivity in neural mass models applied to event-related potentials. Neuroimage 108, 364-376. doi: 10.1016/j.neuroimage.2014.12.067

Zhdanov, A., Nurminen, J., Baess, P., Hirvenkari, L., Jousmaki, V., Makela, J. P., et al. (2015). An internet-based real-time audiovisual link for dual MEG recordings. PLoS ONE 10:e0128485. doi: 10.1371/journal.pone.0128485

Zhu, J., Bellanger, J. J., Shu, H. Z., Yang, C. F., and Jeannes, R. L. B. (2014). Bias reduction in the estimation of mutual information. Phys. Rev. E 90:052714. doi: $10.1103 /$ physreve.90.052714

Conflict of Interest Statement: The authors declare that the research was conducted in the absence of any commercial or financial relationships that could be construed as a potential conflict of interest.

Copyright (c) 2016 Kida, Tanaka and Kakigi. This is an open-access article distributed under the terms of the Creative Commons Attribution License (CC BY). The use, distribution or reproduction in other forums is permitted, provided the original author(s) or licensor are credited and that the original publication in this journal is cited, in accordance with accepted academic practice. No use, distribution or reproduction is permitted which does not comply with these terms. 